\title{
An approach to Atanassov's interval-valued intuitionistic fuzzy multi-attribute decision making based on prospect theory
}

\author{
Fanyong Meng ${ }^{\mathrm{a}, \mathrm{b}}$ * \\ ${ }^{a}$ School of Business, Central South University, Changsha 410083, China \\ ${ }^{b}$ School of Management, Qingdao Technological University, Qingdao 266520, China \\ Chunqiao Tan \\ School of Business, Central South University, Changsha 410083, China \\ Xiaohong Chen \\ School of Business, Central South University, Changsha 410083, China
}

Received 3 June 2014

Accepted 10 March 2015

\begin{abstract}
Prospect theory is a very effective method to express behavioral decision making under uncertainty. This paper attempts to develop a method to multi-attribute decision making with Atanassov's interval-valued intuitionistic fuzzy information using prospect theory. This method first transforms Atanassov's interval-valued intuitionistic fuzzy variables into the prospect values using the value function in prospect theory. Based on the aspiration levels, Atanassov's intuitionistic fuzzy prospect gain and loss matrices are obtained. Then, using the Atanassov's interval-valued intuitionistic hybrid weight averaging (IVIHWA) operator or the Atanassov's interval-valued intuitionistic hybrid Shapley weight averaging (IVIHSWA) operator, the comprehensive Atanassov's interval-valued intuitionistic fuzzy prospect value of each alternative is calculated. According to the comprehensive Atanassov's interval-valued intuitionistic fuzzy prospect values, a ranking method of alternatives is presented. Finally, two illustrative examples are selected to show the feasibility and availability of the proposed method.
\end{abstract}

Keywords: multi-attribute decision making; prospect theory; Atanassov's interval-valued intuitionistic fuzzy set; Atanassov's interval-valued intuitionistic fuzzy prospect value

\section{Introduction}

Because of the highly complex social and economic environment, the decision information of alternatives is usual incompletely known. Hence, fuzzy decision making has become a hot research point and has achieved many important results [1-7]. Atanassov's interval-valued intuitionistic fuzzy sets (IVIFSs) [8], as an important extending form of Zadeh's fuzzy sets, have received considerable attentions, which are characterized by an interval membership function and an interval non-membership function. In 1994, Atanassov [9] presented some operations on IVIFSs. After that, many Atanassov's interval-valued intuitionistic fuzzy aggregation operators are presented [10-15]. As Beliakov et al. [16] pointed for Atanassov's intuitionistic fuzzy numbers (IFNs), there are some undesirable properties of the opera-

*Corresponding author. Tel.: +86-18254298903; Fax: +86-0532-86875851.

E-mail address: mengfanyongtjie@163.com. 
tional laws on IVIFSs [17] as well as the ranking method for the score and accuracy functions [18].

Besides the aggregation operators on IVIFSs, many researchers devoted themselves to decision making with Atanassov's interval-valued intuitionistic fuzzy information [19-23] and applied the Atanassov's interval-valued intuitionistic fuzzy theory in many fields [24-28]. Although many multi-attribute decision making approaches with under Atanassov's interval-valued intuitionistic fuzzy information are researched, none of them consider the decision makers' risk attitudes. Prospect theory proposed by Kahneman and Tversky [29] seems to well deal with this issue, which has become one of the most important descriptive alternatives to expectation utility theory. The rationality of prospect theory was widely supported by experimentations. The core of prospect theory is the value function, which shows the risk aversion for gains, and reflects the risk propensity for losses [30]. Prospect theory has been successfully applied the behavioral decision-making model [30-35]. For the moment, there are few researches about the application of prospect theory to Atanassov's interval-valued intuitionistic fuzzy multi-attribute decision making problems. The purpose of this paper is to analyze a method to multi-attribute decision making with Atanassov's interval-valued intuitionistic fuzzy information problems using prospect theory, which considers the decision makers' attitudes for gains and losses. Based on the Atanassov's interval-valued intuitionistic hybrid weight averaging (IVIHWA) operator or the interval-valued Atanassov's hybrid Shapley weight averaging (IVIHSWA) operator, the comprehensive attribute Atanassov's interval-valued intuitionistic fuzzy prospect values are obtained. Then, a ranking method is introduced.

This article is organized as follows: In section 2, some basic notions are briefly reviewed. In section 3 , the gain and loss IVIFN decision matrices are obtained by calculating gain and loss IVIFNs of every alternative for the reference point. Based on the value function in prospect theory, the Atanassov's interval-valued intuitionistic fuzzy prospect gain and loss matrices are got, whose elements are Atanassov's interval-valued intuitionistic fuzzy prospect values. Then, the IVIHWA and IVIHSWA operators are defined to aggregate Atanassov's interval-valued intuitionistic fuzzy prospect values. Furthermore, a method to multi-attribute decision making with Atanassov's interval-valued intuitionistic fuzzy information based on prospect theory is developed. In section 4 , the associated examples are selected to illustrate the availability of the procedure method.

\section{Some basic concepts}

\subsection{Atanassov's interval-valued intuitionistic fuzzy sets}

In order to overcome the drawback of Zadeh's fuzzy sets that only give us the membership information, Atanassov and Gargov [8] presented the concept of IVIFSs, which do not only provide us the membership information, but also offer the non-membership information and the accuracy information.

Definition 2.1 [8] Let $X$ be a no empty finite set. An IVIFS $A$ in $X$ is expressed as

$$
A=\{\langle x,[a(x), b(x)],[c(x), d(x)]\rangle \mid x \in X\},
$$

where $[a(x), b(x)] \subseteq[0,1]$ and $[c(x), d(x)] \subseteq[0,1]$ are the interval membership and non-membership degrees of the element $x \in X$ with the condition $b(x)+d(x) \leq 1$.

When $a(x)=b(x)$ and $c(x)=d(x)$ for each $x \in X$, then the IVIFS $A$ degenerates to an Atanassov's intuitionistic fuzzy set (IFS) [36], denoted by $A=\{\langle x, a(x), c(x)\rangle \mid x \in X\}$. For convenience of discussion, $\mathrm{Xu}$ [17] gave the definition of IVIFNs: An IVIFN $\tilde{v}$ is defined by $\tilde{v}=([a, b],[c, d])$, where $[a, b] \subseteq[0,1]$ and $[c, d] \subseteq[0,1]$ are respectively the interval membership and non-membership degrees with the condition $b+d \leq 1$.

Similar to the operations on Atanassov's intuitionistic fuzzy sets [37], Xu [17] defined the following operations on IVIFNs.

Definition 2.2 [17] For any two IVIFNs $\tilde{v}=([a, b],[c, d])$ and $\tilde{\gamma}=([e, f],[g, h]), \quad$ the following operations are defined

(1) $\tilde{v} \oplus \tilde{\gamma}=([a+e-a e, b+f-b f],[c g, d h])$;

(2) $\tilde{v} \otimes \tilde{\gamma}=([a e, b f],[c+g-c g, d+h-d h])$;

(3) $\lambda \tilde{v}=\left(\left[1-(1-a)^{\lambda}, 1-(1-b)^{\lambda}\right],\left[c^{\lambda}, d^{\lambda}\right]\right)$ $\lambda \in[0,1]$;

(4) $\tilde{v}^{\lambda}=\left(\left[a^{\lambda}, b^{\lambda}\right],\left[1-(1-c)^{\lambda}, 1-(1-d)^{\lambda}\right]\right)$ $\lambda \in[0,1]$.

In order to research the application of IVIFNs, $\mathrm{Xu}$ and Chen [18] presented the score function $s(\tilde{v})=0.5(a-c+b-d)$ and the accuracy function $h(\tilde{v})=0.5(a+c+b+d)$ to obtain the score and the 
accuracy degree of any IVIFN $\tilde{v}=([a, b],[c, d])$. The order relationship, for any two IVIFNs $\tilde{v}$ and $\tilde{\gamma}$, is defined by

$$
\begin{aligned}
& \text { If } s(\tilde{v})<s(\tilde{\gamma}) \text {, then } \tilde{v}<\tilde{\gamma} . \\
& \text { If } s(\tilde{v})=s(\tilde{\gamma}) \text {, then }\left\{\begin{array}{l}
h(\tilde{v})=h(\tilde{\gamma}) \Rightarrow \tilde{v}=\tilde{\gamma} \\
h(\tilde{v})<h(\tilde{\gamma}) \Rightarrow \tilde{v}<\tilde{\gamma}
\end{array}\right.
\end{aligned}
$$

As Beliakov et al. [16] pointed for IFNs, there exist some undesirable properties of the operational laws on IVIFSs [17] as well as the ranking method for the score and accuracy functions [18], such as the operations (3) and (4) do not preserve the above given order relationship under multiplication or exponentiation by a scalar: $\tilde{v} \prec \tilde{\gamma}$ cannot guarantee $\lambda \tilde{v} \prec \lambda \tilde{\gamma}$ or $\tilde{v}^{\lambda} \prec \tilde{\gamma} \lambda$ for $\lambda \in[0,1]$. Furthermore, it has $\tilde{v} \oplus \tilde{\gamma}=([1,1],[0,0])$ if the interval membership and non-membership degrees of the IVIFN $\tilde{v}$ are equal to $[1,1]$ and $[0,0]$, and it has $\tilde{v} \otimes \tilde{\gamma}=([0,0],[1,1])$ if the interval membership and non-membership degrees of the IVIFN $\tilde{v}$ are equal to $[0,0]$ and $[1,0]$, which are rather counterintuitive. Especially, these issues exist in the aggregation operators [10-15].

Similar to the operations on IFNs [16], for any two IVIFNs $\tilde{v}$ and $\tilde{\gamma}$, we adopt the following operations on IVIFNs:

(I) If there exists IVIFN $\tilde{\beta}$ such that $\tilde{\beta}=\tilde{v} \oplus \tilde{\gamma}$, $\tilde{v} \oplus \tilde{\gamma}=([a+e, b+f],[c+g, d+h]) ;$

(II) $\tilde{v} \otimes \tilde{\gamma}=([a e, b f],[c g, d h])$;

(III) $\lambda \tilde{v}=([\lambda a, \lambda b],[\lambda c, \lambda d]) \quad \lambda \in[0,1]$;

(IV) $\tilde{v}^{\lambda}=\left(\left[a^{\lambda}, b^{\lambda}\right],\left[c^{\lambda}, d^{\lambda}\right]\right) \quad \lambda \in[0,1]$.

\subsection{Prospect theory}

Prospect theory, as the most popular behavioral decision theory, was initially introduced by Kahneman and Tversky [29]. In prospect theory, the value function plays a key role, which owns the following characteristics: (i) The definition domain is deviation from the given reference point; (ii) For gains, it is a concave function, and for losses, it is a convex function; (iii) it is more gently for gains than for losses. Kahneman and Tversky [29] introduced the following explicit expression of the value function

$$
v(x)=\left\{\begin{array}{ll}
x^{\alpha} & x \geq 0 \\
-\lambda(-x)^{\beta} & x<0
\end{array},\right.
$$

where $\alpha$ and $\beta$, respectively, denote parameters for gains and losses, $0 \leq \alpha, \beta \leq 1$. The parameter $\lambda$ represents the steep degree for losses such that $\lambda>1$ for risk aversion.

Kahneman and Tversky [29] considered $\alpha=\beta=0.88$, and $\lambda=2.25$, which are obtained by experimentally data. Abdellaoui [38] suggested that the values of $\alpha$ and $\beta$ equal 0.89 and 0.92 , respectively. Furthermore, Kallneman and Tversky [29] suggested that $\lambda$ belongs to $[2,2.5]$.

In view of the above characteristics of the value function, it can be described by an S-shaped function as illustrated in Fig. 1.

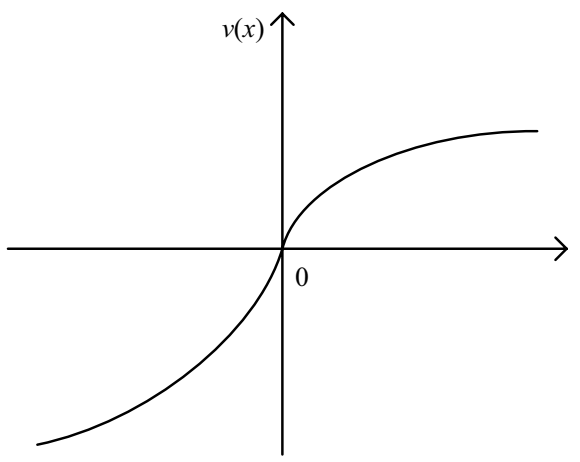

Fig.1. The value function

\section{A method to multi-attribute decision making under Atanassov's interval-valued intuitionistic fuzzy environment based on prospect theory}

This section develops a method to multi-attribute decision making based on prospect theory, where the alternative values and the attribute preference points are IVIFNs. First, let us consider the so-called Atanassov's interval-valued intuitionistic fuzzy prospect gain and loss matrices.

\subsection{Atanassov's interval-valued intuitionistic fuzzy prospect gain and loss matrices}

Let us review the difference on interval numbers. $\bar{a}=\left[a^{-}, a^{+}\right]$is called an interval number if $a^{-} \leq a^{+}$, where $a^{-}, a^{+} \in \mathfrak{R}$, and $\bar{a}=\left[a^{-}, a^{+}\right]$is called a positive interval number if $a^{-} \leq a^{+}$, where $a^{-}, a^{+} \in \mathfrak{R}^{+}$. All interval numbers are denoted by $\overline{\mathfrak{R}}$. 
Let $\bar{a}, \bar{b} \in \overline{\mathfrak{R}}$, from Minkowski operations on intervals, it has

(1) $\bar{a}+\bar{b}=\left[a^{-}+b^{-}, a^{+}+b^{+}\right]$,

(2) $\bar{a}-\bar{b}=\left[a^{-}-b^{+}, a^{+}-b^{-}\right]$,

(3) $\lambda \bar{a}=\left[\lambda a^{-}, \lambda a^{+}\right] \quad \lambda \in \mathfrak{R}_{+}$.

In general, we cannot have $\bar{a}+\bar{b}-\bar{b}=\bar{a}$ for all $\bar{a}, \bar{b} \in \overline{\mathfrak{R}}$. The Hukuhara difference on fuzzy sets [39] can cope with this issue.

Definition 3.1 For any two interval numbers $\bar{a}$ and $\bar{b}$, if there exists $\bar{c} \in \overline{\mathfrak{R}}$ with $\bar{a}=\bar{b}+\bar{c}$, then $\bar{c}$ is called the Hukuhara difference between $\bar{a}$ and $\bar{b}$, expressed by $\bar{a}-{ }_{H} \bar{b}$.

From Definition 3.1, we know $\bar{a}-_{H} \bar{a}=0$ and $\bar{a}+\bar{b}-{ }_{H} \bar{b}=\bar{a}-{ }_{H} \bar{b}+\bar{b}=\bar{a}$ for all $\bar{a}, \bar{b} \in \overline{\mathfrak{R}}$. Later, Stefanini [40] proposed the generalized Hukuhara difference as follows:

$$
\bar{a}-{ }_{g H} \bar{b}=\bar{c} \Leftrightarrow\left\{\begin{array}{c}
\text { (i) }\left\{\begin{array}{l}
a^{-}=b^{-}+c^{-} \\
a^{+}=b^{+}+c^{+}
\end{array}\right. \\
\operatorname{or}(\mathrm{ii})\left\{\begin{array}{l}
b^{-}=a^{-}-c^{+} \\
b^{+}=a^{+}-c^{-}
\end{array}\right.
\end{array}\right.
$$

where $\bar{a}=\left[a^{-}, a^{+}\right], \bar{b}=\left[b^{-}, b^{+}\right], \bar{c}=\left[c^{-}, c^{+}\right] \in \overline{\mathfrak{R}}$.

Eq.(2) is equivalent to $\bar{a}-{ }_{g H} \bar{b}=\bar{c}$ with

$$
c^{-}=\min \left\{a^{-}-b^{-}, a^{+}-b^{+}\right\}
$$

and

$$
c^{+}=\max \left\{a^{-}-b^{-}, a^{+}-b^{+}\right\} .
$$

More details can be seen in [40]. In this paper, without special explanation, we will adopt the generalized Hukuhara difference on interval numbers.

Form Eq.(1), it is easy to drive that the value function in prospect theory defined on interval numbers can be expressed by

$$
v(x)=\left\{\begin{array}{c}
{\left[a^{\alpha}, b^{\alpha}\right] \quad x \in[a, b], a \geq 0} \\
{\left[-\lambda(-a)^{\beta},-\lambda(-b)^{\beta}\right] \quad x \in[a, b], b \leq 0}
\end{array} .\right.
$$

Consider a multi-attribute decision making problem, without loss of generality, suppose there are $m$ alternatives $A=\left\{a_{1}, a_{2}, \ldots, a_{m}\right\}$ and $n$ attributes $C=\left\{c_{1}, c_{2}, \ldots, c_{n}\right\}$. By $\tilde{A}=\left(\tilde{v}_{i j}\right)_{m \times n}$, we denote the IVIFN decision matrix given by the experts, where $\tilde{v}_{i j}=\left(\left[a_{i j}, b_{i j}\right],\left[c_{i j}, d_{i j}\right]\right)(i=1,2, \ldots, m ; j=1,2, \ldots, n)$ is an IVIFN with $\left[a_{i j}, b_{i j}\right] \subseteq[0,1]$ and $\left[c_{i j}, d_{i j}\right] \subseteq[0,1]$ being the interval membership and non-membership degrees of the alternative $a_{i}$ with respect to the attribute $c_{j}$. Let $\tilde{S}=\left(\tilde{s}_{1}, \tilde{s}_{2}, \ldots, \tilde{s}_{n}\right)$ be the IVIFN reference vector given by the experts, where $\tilde{s}_{j}=\left(\left[u_{l}^{j}, u_{r}^{j}\right],\left[v_{l}^{j}, v_{r}^{j}\right]\right)$ is the IVIFN reference point of the alternatives with respect to the attribute $c_{j}$.

Without loss of generality, assume that $c_{j}$ is a benefit attribute; otherwise, we can transform it into a benefit attribute [41]. With respect to the IVIFNs $\tilde{v}_{i j}$ and $\tilde{s}_{j}$, since the generalized Hukuhara difference $\left[a_{i j}, b_{i j}\right]-_{g H}\left[u_{l}^{j}, u_{r}^{j}\right]$ between the interval membership degrees $\left[a_{i j}, b_{i j}\right]$ and $\left[u_{l}^{j}, u_{r}^{j}\right]$ as well as the generalized Hukuhara difference $\left[v_{l}^{j}, v_{r}^{j}\right]-_{g H}$ $\left[c_{i j}, d_{i j}\right]$ between the interval non-membership degrees $\left[v_{l}^{j}, v_{r}^{j}\right]$ and $\left[c_{i j}, d_{i j}\right]$ the bigger the better, $\left(\left[a_{i j}, b_{i j}\right]-_{g H}\left[u_{l}^{j}, u_{r}^{j}\right],\left[v_{l}^{j}, v_{r}^{j}\right]-_{g H}\left[c_{i j}, d_{i j}\right]\right)$ can be seen as the better "IVIFN" than the IVIFN reference point $\tilde{s}_{j}$. Thus, $\tilde{\theta}_{i j}=\left(\left[k_{i j}, y_{i j}\right],\left[t_{i j}, z_{i j}\right]\right)$ can be seen as the gain IVIFN of the alternative $a_{i}$ with respect to the attribute $c_{j}$, where

$$
\begin{aligned}
{\left[k_{i j}, y_{i j}\right]=} & {\left[\max \left\{0, \min \left\{a_{i j}-u_{l}^{j}, b_{i j}-u_{r}^{j}\right\}\right\},\right.} \\
& \left.\max \left\{0, \max \left\{a_{i j}-u_{l}^{j}, b_{i j}-u_{r}^{j}\right\}\right\}\right], \\
{\left[t_{i j}, z_{i j}\right]=[} & \max \left\{0, \min \left\{v_{l}^{j}-c_{i j}, v_{r}^{j}-d_{i j}\right\}\right\}, \\
& \left.\max \left\{0, \max \left\{v_{l}^{j}-c_{i j}, v_{r}^{j}-d_{i j}\right\}\right\}\right] .
\end{aligned}
$$

On the other hand, with respect to the IVIFNs $\tilde{v}_{i j}$ and $\tilde{s}_{j}$, since the generalized Hukuhara difference $\left[u_{l}^{j}, u_{r}^{j}\right]-_{g H}\left[a_{i j}, b_{i j}\right]$ between the interval membership degrees $\left[u_{l}^{j}, u_{r}^{j}\right]$ and $\left[a_{i j}, b_{i j}\right]$ as well as the generalized Hukuhara difference $\left[c_{i j}, d_{i j}\right]-_{g H}\left[v_{l}^{j}, v_{r}^{j}\right]$ between the interval non-membership degrees $\left[c_{i j}, d_{i j}\right]$ and $\left[v_{l}^{j}, v_{r}^{j}\right]$ the smaller the better, $\left(\left[u_{l}^{j}, u_{r}^{j}\right]-_{g H}\left[a_{i j}, b_{i j}\right]\right.$, $\left.\left[c_{i j}, d_{i j}\right]-_{g H}\left[v_{l}^{j}, v_{r}^{j}\right]\right)$ can be regarded as the worse "IVIFN" than the IVIFN reference point $\tilde{s}_{j}$. Hence, $\tilde{\zeta}_{i j}=\left(\left[o_{i j}, p_{i j}\right],\left[q_{i j}, r_{i j}\right]\right)$ can be considered as the loss IVIFN of the alternative $a_{i}$ with respect to the attribute $c_{j}$, where

$$
\begin{aligned}
{\left[o_{i j}, p_{i j}\right]=} & {\left[\max \left\{0, \min \left\{u_{l}^{j}-a_{i j}, u_{r}^{j}-b_{i j}\right\}\right\},\right.} \\
& \left.\max \left\{0, \max \left\{u_{l}^{j}-a_{i j}, u_{r}^{j}-b_{i j}\right\}\right\}\right], \\
{\left[q_{i j}, r_{i j}\right]=} & {\left[\max \left\{0, \min \left\{c_{i j}-v_{l}^{j}, d_{i j}-v_{r}^{j}\right\}\right\},\right.} \\
& \left.\max \left\{0, \max \left\{c_{i j}-v_{l}^{j}, d_{i j}-v_{r}^{j}\right\}\right\}\right] .
\end{aligned}
$$


By $\tilde{G}=\left(\tilde{\theta}_{i j}\right)_{m \times n}$ and $\tilde{L}=\left(\tilde{\zeta}_{i j}\right)_{m \times n}$, we respectively denote the gain and loss IVIFN decision matrices.

From Eq.(3), it gets the Atanassov's intervalvalued intuitionistic fuzzy prospect gain matrix $\nabla G=\left(\tilde{\tau}_{i j}\right)_{m \times n}$ with $\tilde{\tau}_{i j}=\left(\left[k_{i j}{ }^{\alpha}, y_{i j}{ }^{\alpha}\right],\left[t_{i j}{ }^{\alpha}, z_{i j}{ }^{\alpha}\right]\right)$ and the Atanassov's interval-valued intuitionistic fuzzy prospect loss matrix $\forall L=\left(\tilde{\pi}_{i j}\right)_{m \times n} \quad$ with $\tilde{\pi}_{i j}=\left(\left[-\lambda p_{i j}{ }^{\beta},-\lambda o_{i j}{ }^{\beta}\right],\left[-\lambda r_{i j}^{\beta},-\lambda q_{i j}{ }^{\beta}\right]\right)$.

Remark 3.1 The elements in the so-called Atanassov's interval-valued intuitionistic fuzzy prospect matrix can be any real number. Here, $\nabla_{G}$ and $V L$ are called Atanassov's interval-valued intuitionistic fuzzy prospect gain and loss matrices just because their elements are obtained from the IVIFN decision matrix $\tilde{A}=\left(\tilde{v}_{i j}\right)_{m \times n}$.

If $a_{i j}=b_{i j}$ and $c_{i j}=d_{i j}$ for all $i=1,2, \ldots, m ; j=1$, $2, \ldots, n$, then the IVIFN decision matrix $\tilde{A}$ degenerates to the IFN decision matrix $\tilde{A}^{\prime}=\left(\tilde{v}_{i j}^{\prime}\right)_{m \times n}$, where $\tilde{v}_{i j}^{\prime}=\left(a_{i j}, c_{i j}\right)(i=1,2, \ldots, m ; j=1,2, \ldots, n)$ is an IFN with $a_{i j} \in[0,1]$ and $c_{i j} \in[0,1]$ being the interval membership and non-membership degrees of the alternative $a_{i}$ with respect to the attribute $c_{j}$. Let $\tilde{S}^{\prime}=\left(\tilde{s}_{1}^{\prime}, \tilde{S}_{2}, \ldots, \tilde{S}_{n}{ }_{n}\right)$ be the IFN reference vector given by the experts, where $\tilde{s}^{\prime}{ }_{j}=\left(u_{j}, v_{j}\right)$ is the IFN reference point of the alternatives with respect to the attribute $c_{j}$.

As was analyzed above, it gets the gain IFN decision matrix $\tilde{G}^{\prime}=\left(\tilde{\theta}^{\prime}{ }_{i j}\right)_{m \times n}$, where $\tilde{\theta}_{i j}^{\prime}=\left(k_{i j}, t_{i j}\right)$ with $k_{i j}=\max \left\{0, a_{i j}-u_{j}\right\}$ and $t_{i j}=\max \left\{0, v_{l}^{j}-c_{i j}\right\}$, and the loss IFN decision matrix $\tilde{L}^{\prime}=\left(\tilde{\zeta}^{\prime}{ }_{i j}\right)_{m \times n}$, where $\tilde{\zeta}_{i j}^{\prime}=\left(o_{i j}, q_{i j}\right)$ with $o_{i j}=\max \left\{0, u_{j}-a_{i j}\right\}$ and $q_{i j}=\max \left\{0, c_{i j}-v_{l}^{j}\right\}$. Furthermore, Atanassov's intuitionistic fuzzy prospect gain and loss matrices are respectively obtained by

and

$$
\nabla G^{\prime}=\left(\tilde{\tau}^{\prime}{ }_{i j}\right)_{m \times n}=\left(\left(k_{i j}{ }^{\alpha}, t_{i j}{ }^{\alpha}\right)\right)_{m \times n}
$$

$$
\mathscr{V} L^{\prime}=\left(\tilde{\pi}_{i j}^{\prime}\right)_{m \times n}=\left(\left(-\lambda o_{i j}{ }^{\beta},-\lambda q_{i j}{ }^{\beta}\right)\right)_{m \times n} .
$$

For any IVIFN $\tilde{v}$ and the given IVIFN preference point $\tilde{\zeta}$, assume that $\tilde{\tau}=\left(\left[u_{l}^{G}, u_{r}^{G}\right],\left[v_{l}^{G}, v_{r}^{G}\right]\right)$ and
$\tilde{\pi}=\left(\left[u_{l}^{L}, u_{r}^{L}\right],\left[v_{l}^{L}, v_{r}^{L}\right]\right)$ are Atanassov's intervalvalued intuitionistic fuzzy prospect gain and loss values with respect to $\tilde{v}$ and $\tilde{\zeta}$.

The absolute index is defined by

$$
A I(\tilde{\tau}, \tilde{\pi})=u_{l}^{G}+u_{r}^{G}+v_{l}^{G}+v_{r}^{G}+u_{l}^{L}+u_{r}^{L}+v_{l}^{L}+v_{r}^{L},
$$

and the relative index is expressed by

$$
R I(\tilde{\tau}, \tilde{\pi})=\frac{u_{l}^{G}+u_{r}^{G}+v_{l}^{G}+v_{r}^{G}}{u_{l}^{G}+u_{r}^{G}+v_{l}^{G}+v_{r}^{G}-\left(u_{l}^{L}+u_{r}^{L}+v_{l}^{L}+v_{r}^{L}\right)} .
$$

Let $\tilde{v}_{1}$ and $\tilde{v}_{2}$ be any two IVIFNs, and $\tilde{\zeta}$ be the IVIFN preference point. Assume that

$$
\tilde{\tau}_{i}=\left(\left[u_{i l}^{G}, u_{i r}^{G}\right],\left[v_{i l}^{G}, v_{i r}^{G}\right]\right)
$$

and

$$
\tilde{\pi}_{i}=\left(\left[u_{i l}^{L}, u_{i r}^{L}\right],\left[v_{i l}^{L}, v_{i r}^{L}\right]\right)
$$

are Atanassov's interval-valued intuitionistic fuzzy prospect gain and loss values with respect to $\tilde{v}_{i}$ and $\tilde{\zeta}$, $i=1,2$. Then, their order relationship is defined by

i) If $A I\left(\tilde{\tau}_{1}, \tilde{\pi}_{1}\right) \leq A I\left(\tilde{\tau}_{2}, \tilde{\pi}_{2}\right)$ or $A I\left(\tilde{\tau}_{1}, \tilde{\pi}_{1}\right)=A I\left(\tilde{\tau}_{2}, \tilde{\pi}_{2}\right)$ and $R I\left(\tilde{\tau}_{1}, \tilde{\pi}_{1}\right) \leq R I\left(\tilde{\tau}_{2}, \tilde{\pi}_{2}\right)$, then $\tilde{v}_{1} \leq \tilde{v}_{2}$;

ii) If $A I\left(\tilde{\tau}_{1}, \tilde{\pi}_{1}\right)=A I\left(\tilde{\tau}_{2}, \tilde{\pi}_{2}\right)$ and $\operatorname{RI}\left(\tilde{\tau}_{1}, \tilde{\pi}_{1}\right)=\operatorname{RI}\left(\tilde{\tau}_{2}, \tilde{\pi}_{2}\right)$, then $\tilde{v}_{1}=\tilde{v}_{2}$.

\subsection{The IVIHWA and IVIHSWA operators}

Based on the new defined operations on IFNs, Beliakov et al. [16] introduced the IWAM operator.

Let $\tilde{v}_{j}=\left(e_{j}, f_{j}\right) \quad(j=1,2, \ldots, n)$ be a collection of IFNs, and $\omega=\left(\omega_{1}, \omega_{2}, \ldots, \omega_{n}\right)$ be the weight vector, then the IWAM operator is defined by

$$
\operatorname{IWAM}_{\omega}\left(\tilde{v}_{1}, \tilde{v}_{2}, \ldots, \tilde{v}_{n}\right)=\left(\sum_{j=1}^{n} \omega_{j} e_{j}, \sum_{j=1}^{n} \omega_{j} f_{j}\right) .
$$

Similar to the IWAM operator, we introduce the following IVIHWA operator, by which the comprehensive Atanassov's interval-valued intuitionistic fuzzy prospect gain and loss values can be obtained. It considers the importance of elements and their ordered positions.

Definition 3.2 An IVIHWA operator of dimension $n$ is a mapping IVIHWA: IVIFNs ${ }^{n} \rightarrow$ IVIFNs with the associated weight vector $w=\left(w_{1}, w_{2}, \ldots, w_{n}\right)$ on the ordered set $N=\{1,2, \ldots, n\}$ being $w_{j} \in[0,1]$ and $\sum_{j=1}^{n} w_{j}=1$, denoted by

$$
\operatorname{IVIHWA}_{w, \omega}\left(\tilde{v}_{1}, \tilde{v}_{2}, \ldots, \tilde{v}_{n}\right)=\frac{\sum_{j=1}^{n} w_{j} \omega_{(j)} \tilde{v}_{(j)}}{\sum_{j=1}^{n} w_{j} \omega_{(j)}}
$$




$$
\begin{gathered}
=\left(\left[\frac{\sum_{j=1}^{n} w_{j} \omega_{(j)} a_{(j)}}{\sum_{j=1}^{n} w_{j} \omega_{(j)}}, \frac{\sum_{j=1}^{n} w_{j} \omega_{(j)} b_{(j)}}{\sum_{j=1}^{n} w_{j} \omega_{(j)}}\right],\right. \\
\left.\left[\frac{\sum_{j=1}^{n} w_{j} \omega_{(j)} c_{(j)}}{\sum_{j=1}^{n} w_{j} \omega_{(j)}}, \frac{\sum_{j=1}^{n} w_{j} \omega_{(j)} d_{(j)}}{\sum_{j=1}^{n} w_{j} \omega_{(j)}}\right]\right),
\end{gathered}
$$

where (') is a permutation on $N$ such that $\tilde{v}_{(j)}$ being the $j$ th largest value of $\tilde{v}_{i}=\left(\left[a_{i}, b_{i}\right],\left[c_{i}, d_{i}\right]\right)$, and $\omega=\left(\omega_{1}, \omega_{2}, \ldots, \omega_{n}\right)$ is the weight vector on $\tilde{v}_{i}(i=1$, $2, \ldots, n)$ with $\omega_{i}>0$ and $\sum_{i=1}^{n} \omega_{i}=1$.

Remark 3.2 From the expression of the IVIHWA operator, it is easy to see that if $w_{j}=1 / n$ for all $j=1$, $2, \ldots, n$, then it gets the Atanassov's interval-valued intuitionistic weight averaging (IVIWA) operator

$$
\operatorname{IVIHWA}_{\omega}\left(\tilde{v}_{1}, \tilde{v}_{2}, \ldots, \tilde{v}_{n}\right)=\sum_{j=1}^{n} \omega_{j} \tilde{v}_{j}
$$

$$
=\left(\left[\sum_{j=1}^{n} \omega_{j} a_{j}, \sum_{j=1}^{n} \omega_{j} b_{j}\right],\left[\sum_{j=1}^{n} \omega_{j} c_{j}, \sum_{j=1}^{n} \omega_{j} d_{j}\right]\right) \text {. }
$$

Furthermore, if $\omega_{i}=1 / n$ for all $i=1,2, \ldots, n$, then it gets the Atanassov's interval-valued intuitionistic ordered weight averaging (IVIOWA) operator

$$
\begin{aligned}
& \text { IVIOWA }_{w}\left(\tilde{v}_{1}, \tilde{v}_{2}, \ldots, \tilde{v}_{n}\right)=\sum_{j=1}^{n} w_{j} \tilde{v}_{(j)} \\
& =\left(\left[\sum_{j=1}^{n} w_{j} a_{(j)}, \sum_{j=1}^{n} w_{j} b_{(j)}\right],\left[\sum_{j=1}^{n} w_{j} c_{(j)}, \sum_{j=1}^{n} w_{j} d_{(j)}\right]\right),
\end{aligned}
$$

where (') is a permutation on $N$ such that $\tilde{v}_{(j)}$ being the $j$ th largest value of $\tilde{v}_{i}=\left(\left[a_{i}, b_{i}\right],\left[c_{i}, d_{i}\right]\right)$.

If each IVIFN $\tilde{v}_{i}(i=1,2, \ldots, n)$ degenerates to be IFN $\tilde{v}_{i}=\left(e_{i}, f_{i}\right)$, then it gets the Atanassov's intuitionistic hybrid weight averaging (IHWA) operator

$$
\begin{gathered}
\operatorname{IHWA}_{w, \omega}\left(\tilde{v}_{1}, \tilde{v}_{2}, \ldots, \tilde{v}_{n}\right)=\frac{\sum_{j=1}^{n} w_{j} \omega_{(j)} \tilde{v}_{(j)}}{\sum_{j=1}^{n} w_{j} \omega_{(j)}} \\
=\left(\frac{\sum_{j=1}^{n} w_{j} \omega_{(j)} e_{(j)}}{\sum_{j=1}^{n} w_{j} \omega_{(j)}}, \frac{\sum_{j=1}^{n} w_{j} \omega_{(j)} f_{(j)}}{\sum_{j=1}^{n} w_{j} \omega_{(j)}}\right),
\end{gathered}
$$

where (') is a permutation on $N$ such that $\tilde{v}_{(j)}$ being the $j$ th largest value of $\tilde{v}_{i}=\left(e_{i}, f_{i}\right), i=1,2, \ldots, n$.

Furthermore, if $w_{j}=1 / n$ for all $j=1,2, \ldots, n$, then the IHWA operator reduces to be the IWAA operator [16], and if $\omega_{i}=1 / n$ for all $i=1,2, \ldots, n$, then the IHWA operator degenerates to be the Atanassov's intuitionistic ordered weight averaging (IOWA) operator

$$
\begin{aligned}
& \operatorname{IOWA}_{w}\left(\tilde{v}_{1}, \tilde{v}_{2}, \ldots, \tilde{v}_{n}\right)=\sum_{j=1}^{n} w_{j} \tilde{v}_{(j)} \\
& =\left(\sum_{j=1}^{n} w_{j} e_{(j)}, \sum_{j=1}^{n} w_{j} f_{(j)}\right),
\end{aligned}
$$

where ( ) is a permutation on $N$ such that $\tilde{v}_{(j)}$ being the $j$ th largest value of $\tilde{v}_{i}=\left(e_{i}, f_{i}\right)$.

Although the IVIHWA operator both considers the importance of elements and that of the ordered positions, it is based on the assumption that the elements in a set are uncorrelated. Similarly to researches about aggregation operators [42, 43] based on Shapley function, we further define the IVIHSWA operator. First, let us review the expression of the Shapley function [44]

$$
S h_{i}(\mu, N)=\sum_{S \subseteq N \backslash i} \frac{(n-s-1) ! s !}{n !}(\mu(S \bigcup i)-\mu(S))
$$

$$
\forall i \in N,
$$

where $\mu$ is a fuzzy measure [45] on the power set $P(N)$ of $N=\{1,2, \ldots, n\}$, i.e., $\mu: P(N) \rightarrow[0,1]$ such that (i) $\mu(\varnothing)=0, \mu(N)=1$; (ii) For all $A, B \in P(N)$ with $A \subseteq B, \mu(A) \leq \mu(B)$, and $s$ and $n$ are, respectively, the cardinalities of $S$ and $N$.

If there are no interactions between elements in $N$, then $\mu$ is an additive measure, and for any $i \in N$, $S h_{i}(\mu, N)=\mu(i)$. From the properties of the Shapley function, it is easy to know that $\left\{S h_{i}(\mu, N)\right\}_{i \in N}$ is a weight vector on $N$.

Definition 3.5 An IVIHSWA operator of dimension $n$ is a mapping IVIHSWA: IVIFNs ${ }^{n} \rightarrow$ IVIFNs with the fuzzy measure $\mu$ on the ordered set $N=\{1,2, \ldots$, $n\}$ and the fuzzy measure $v$ on $U=\left\{\tilde{v}_{i}\right\}_{i \in N}$, denoted by

$$
\begin{aligned}
& \operatorname{IVIHSWA}_{\mu, v}\left(\tilde{v}_{1}, \tilde{v}_{2}, \ldots, \tilde{v}_{n}\right) \\
& =\frac{\sum_{j=1}^{n} S h_{j}(\mu, N) S h_{\tilde{V}_{(j)}}(v, U) \tilde{v}_{(j)}}{\sum_{j=1}^{n} S h_{j}(\mu, N) S h_{\tilde{v}_{(j)}}(v, U)} \\
& =\left(\left[\frac{\sum_{j=1}^{n} S h_{j}(\mu, N) S h_{\tilde{v}_{(j)}}(v, U) a_{(j)}}{\sum_{j=1}^{n} S h_{j}(\mu, N) S h_{\tilde{v}_{(j)}}(v, U)},\right.\right. \\
& \left.\frac{\sum_{j=1}^{n} S h_{j}(\mu, N) S h_{\tilde{v}_{(j)}}(v, U) b_{(j)}}{\sum_{j=1}^{n} S h_{j}(\mu, N) S h_{\tilde{v}_{(j)}}(v, U)}\right] \text {, } \\
& {\left[\frac{\sum_{j=1}^{n} S h_{j}(\mu, N) S h_{\tilde{v}_{(j)}}(v, U) c_{(j)}}{\sum_{j=1}^{n} S h_{j}(\mu, N) S h_{\tilde{v}_{(j)}}(v, U)},\right.}
\end{aligned}
$$




$$
\left.\left.\frac{\sum_{j=1}^{n} S h_{j}(\mu, N) S h_{\tilde{v}_{(j)}}(v, U) d_{(j)}}{\sum_{j=1}^{n} S h_{j}(\mu, N) S h_{\tilde{v}_{(j)}}(v, U)}\right]\right),
$$

where $S h_{\tilde{v}_{j}}(v, U)$ is the Shapley value of $\tilde{v}_{i}$, () is a permutation on $N$ such that $S h_{\tilde{v}_{(j)}}(v, U) \tilde{v}_{(j)}$ being the $j$ th largest value of $S h_{\tilde{v}_{i}}(v, U) \tilde{v}_{i}$, and $S h_{j}(\mu, N)$ is the Shapley value of the $j$ th position.

Theorem 3.1 Let $\tilde{v}_{i}(i=1,2, \ldots, n)$ be a set of IVIFNs, then their aggregated value obtained from the IVIHSWA operator is still an IVIFN.

Proof. From Definition 3.5, it only needs to show

$$
\begin{aligned}
& {\left[\frac{\sum_{j=1}^{n} S h_{j}(\mu, N) S h_{\tilde{U}_{(j)}}(v, U) a_{(j)}}{\sum_{j=1}^{n} S h_{j}(\mu, N) S h_{\tilde{v}_{(j)}}(v, U)},\right.} \\
& \left.\frac{\sum_{j=1}^{n} S h_{j}(\mu, N) S h_{\tilde{v}_{(j)}}(v, U) b_{(j)}}{\sum_{j=1}^{n} S h_{j}(\mu, N) S h_{\tilde{V}_{(j)}}(v, U)}\right] \subseteq[0,1], \\
& {\left[\frac{\sum_{j=1}^{n} S h_{j}(\mu, N) S h_{\tilde{U}_{(j)}}(v, U) c_{(j)}}{\sum_{j=1}^{n} S h_{j}(\mu, N) S h_{\tilde{U}_{(j)}}(v, U)}\right.} \\
& \left.\frac{\sum_{j=1}^{n} S h_{j}(\mu, N) S h_{\tilde{v}_{(j)}}(v, U) d_{(j)}}{\sum_{j=1}^{n} S h_{j}(\mu, N) S h_{\tilde{U}_{(j)}}(v, U)}\right] \subseteq[0,1]
\end{aligned}
$$

and

$$
\begin{aligned}
& \frac{\sum_{j=1}^{n} S h_{j}(\mu, N) S h_{\tilde{v}_{(j)}}(v, U) b_{(j)}}{\sum_{j=1}^{n} S h_{j}(\mu, N) S h_{\tilde{v}_{(j)}}(v, U)}+ \\
& \frac{\sum_{j=1}^{n} S h_{j}(\mu, N) S h_{\tilde{\nu}_{(j)}}(v, U) d_{(j)}}{\sum_{j=1}^{n} S h_{j}(\mu, N) S h_{\tilde{v}_{(j)}}(v, U)} \leq 1 .
\end{aligned}
$$

From $\left[a_{i}, b_{i}\right] \subseteq[0,1], i=1,2, \ldots, n$, it gets $\left[a_{(j)}, b_{(j)}\right] \subseteq[0,1]$ for all $j=1,2, \ldots, n$. One can easily check that $\left\{\frac{S h_{j}(\mu, N) S h_{\tilde{v}_{(j)}}(v, U)}{\sum_{j=1}^{n} S h_{j}(\mu, N) S h_{\tilde{v}_{(j)}}(v, U)}\right\}_{j=1,2, \ldots, n}$ is a weight vector. Thus,

$$
\begin{gathered}
{\left[\frac{\sum_{j=1}^{n} S h_{j}(\mu, N) S h_{\tilde{v}_{(j)}}(v, U) a_{(j)}}{\sum_{j=1}^{n} S h_{j}(\mu, N) S h_{\tilde{U}_{(j)}}(v, U)},\right.} \\
\left.\frac{\sum_{j=1}^{n} S h_{j}(\mu, N) S h_{\tilde{v}_{(j)}}(v, U) b_{(j)}}{\sum_{j=1}^{n} S h_{j}(\mu, N) S h_{\tilde{U}_{(j)}}(v, U)}\right]
\end{gathered}
$$

$$
\begin{aligned}
& \subseteq \sum_{j=1}^{n} \frac{S h_{j}(\mu, N) S h_{\tilde{V}_{(j)}}(v, U)}{\sum_{j=1}^{n} S h_{j}(\mu, N) S h_{\tilde{v}_{(j)}}(v, U)}\left[a_{(j)}, b_{(j)}\right] \\
& \subseteq \sum_{j=1}^{n} \frac{S h_{j}(\mu, N) S h_{\tilde{V}_{(j)}}(v, U)}{\sum_{j=1}^{n} S h_{j}(\mu, N) S h_{\tilde{V}_{(j)}}(v, U)}[0,1] \\
& =[0,1] .
\end{aligned}
$$

Similarly, one can have

$$
\begin{aligned}
& {\left[\frac{\sum_{j=1}^{n} S h_{j}(\mu, N) S h_{\tilde{v}_{(j)}}(v, U) c_{(j)}}{\sum_{j=1}^{n} S h_{j}(\mu, N) S h_{\tilde{v}_{(j)}}(v, U)},\right.} \\
& \left.\frac{\sum_{j=1}^{n} S h_{j}(\mu, N) S h_{\tilde{v}_{(j)}}(v, U) d_{(j)}}{\sum_{j=1}^{n} S h_{j}(\mu, N) S h_{\tilde{v}_{(j)}}(v, U)}\right] \subseteq[0,1] .
\end{aligned}
$$

Since $b_{(j)}+d_{(j)} \leq 1$ for all $j=1,2, \ldots, n$, it derives

$$
\frac{\sum_{j=1}^{n} S h_{j}(\mu, N) S h_{\tilde{\nu}_{(j)}}(v, U) b_{(j)}}{\sum_{j=1}^{n} S h_{j}(\mu, N) S h_{\tilde{\nu}_{(j)}}(v, U)}+
$$$$
\frac{\sum_{j=1}^{n} S h_{j}(\mu, N) S h_{\tilde{U}_{(j)}}(v, U) d_{(j)}}{\sum_{j=1}^{n} S h_{j}(\mu, N) S h_{\tilde{v}_{(j)}}(v, U)}
$$$$
=\sum_{j=1}^{n} \frac{S h_{j}(\mu, N) S h_{\tilde{v}_{(j)}}(v, U)}{\sum_{j=1}^{n} S h_{j}(\mu, N) S h_{\tilde{v}_{(j)}}(v, U)}\left(b_{(j)}+d_{(j)}\right)
$$$$
\leq \sum_{j=1}^{n} \frac{S h_{j}(\mu, N) S h_{\tilde{v}_{(j)}}(v, U)}{\sum_{j=1}^{n} S h_{j}(\mu, N) S h_{\tilde{v}_{(j)}}(v, U)}=1
$$

Remark 3.3 If there are no interactions between elements $\tilde{v}_{i}$ and the ordered positions, then the IVIHSWA operator degenerates to the IVIHWA operator. If $\operatorname{Sh}_{j}(\mu, N)=1 / n$ for all $j=1,2, \ldots, n$, then it gets the Atanassov's $\tilde{v}_{i}=\left(\left[a_{i}, b_{i}\right],\left[c_{i}, d_{i}\right]\right)$ intervalvalued intuitionistic Shapley weight averaging (IVISWA) operator

$$
\begin{gathered}
\operatorname{IVISWA}_{v}\left(\tilde{v}_{1}, \tilde{v}_{2}, \ldots, \tilde{v}_{n}\right)=\sum_{j=1}^{n} S h_{\tilde{\nu}_{j}}(v, U) \tilde{v}_{j} \\
=\left(\left[\sum_{j=1}^{n} S h_{\tilde{v}_{j}}(v, U) a_{j}, \sum_{j=1}^{n} S h_{\tilde{v}_{j}}(v, U) b_{j}\right],\right. \\
\left.\left[\sum_{j=1}^{n} S h_{\tilde{\nu}_{j}}(v, U) c_{j}, \sum_{j=1}^{n} S h_{\tilde{v}_{j}}(v, U) d_{j}\right]\right) .
\end{gathered}
$$

If $S h_{\tilde{\nu}_{i}}(v, U)=1 / n$ for all $i=1,2, \ldots, n$, then it gets the Atanassov's interval-valued intuitionistic Shapley ordered weight averaging (IVISOWA) operator

$$
\begin{aligned}
& \text { IVISOWA }_{\mu}\left(\tilde{v}_{1}, \tilde{v}_{2}, \ldots, \tilde{v}_{n}\right)=\sum_{j=1}^{n} S h_{j}(\mu, N) \tilde{v}_{(j)} \\
& =\left(\left[\sum_{j=1}^{n} S h_{j}(\mu, N) a_{(j)}, \sum_{j=1}^{n} S h_{j}(\mu, N) b_{(j)}\right],\right. \\
& \left.\quad\left[\sum_{j=1}^{n} S h_{j}(\mu, N) c_{(j)}, \sum_{j=1}^{n} S h_{j}(\mu, N) d_{(j)}\right]\right),
\end{aligned}
$$


where (') is a permutation on $N$ such that $\tilde{v}_{(j)}$ being the $j$ th largest value of $\tilde{v}_{i}=\left(\left[a_{i}, b_{i}\right],\left[c_{i}, d_{i}\right]\right)$.

If each IVIFN $\tilde{v}_{i}(i=1,2, \ldots, n)$ degenerates to be IFN $\tilde{v}_{i}=\left(e_{i}, f_{i}\right)$, then it gets the Atanassov's intuitionistic hybrid Shapley weight averaging (IHSWA) operator

$$
\begin{aligned}
& \operatorname{IHSWA}_{\mu, v}\left(\tilde{v}_{1}, \tilde{v}_{2}, \ldots, \tilde{v}_{n}\right)=\frac{\sum_{j=1}^{n} S h_{j}(\mu, N) S h_{\tilde{v}_{(j)}}(v, U) \tilde{v}_{(j)}}{\sum_{j=1}^{n} S h_{j}(\mu, N) S h_{\tilde{v}_{(j)}}(v, U)} \\
& =\left(\frac{\sum_{j=1}^{n} S h_{j}(\mu, N) S h_{\tilde{v}_{(j)}}(v, U) e_{(j)}}{\sum_{j=1}^{n} S h_{j}(\mu, N) S h_{\tilde{v}_{(j)}}(v, U)},\right. \\
& \left.\frac{\sum_{j=1}^{n} S h_{j}(\mu, N) S h_{\tilde{v}_{(j)}}(v, U) f_{(j)}}{\sum_{j=1}^{n} S h_{j}(\mu, N) S h_{\tilde{v}_{(j)}}(v, U)}\right),
\end{aligned}
$$

where (') is a permutation on $N$ such that $\tilde{v}_{(j)}$ being the $j$ th largest value of $\tilde{v}_{i}=\left(e_{i}, f_{i}\right)$.

Furthermore, if $\operatorname{Sh}_{j}(\mu, N)=1 / n$ for all $j=1,2, \ldots, n$, then the IHSWA operator reduces to be the Atanassov's intuitionistic Shapley weight averaging (ISWA) operator

$$
\begin{aligned}
& \operatorname{ISWA}_{v}\left(\tilde{v}_{1}, \tilde{v}_{2}, \ldots, \tilde{v}_{n}\right)=\sum_{j=1}^{n} S h_{\tilde{v}_{j}}(v, U) \tilde{v}_{j} \\
= & \left(\left[\sum_{j=1}^{n} S h_{\tilde{v}_{j}}(v, U) e_{j}, \sum_{j=1}^{n} S h_{\tilde{v}_{j}}(v, U) f_{j}\right]\right),
\end{aligned}
$$

and if $\operatorname{Sh}_{\tilde{v}_{i}}(v, U)=1 / n$ for all $i=1,2, \ldots, n$, then the IHSWA operator reduces to be the Atanassov's intuitionistic Shapley ordered weight averaging (ISOWA) operator

$$
\begin{aligned}
& \operatorname{ISOWA}_{\mu}\left(\tilde{v}_{1}, \tilde{v}_{2}, \ldots, \tilde{v}_{n}\right)=\sum_{j=1}^{n} S h_{j}(\mu, N) \tilde{v}_{(j)} \\
& =\left(\left[\sum_{j=1}^{n} S h_{j}(\mu, N) e_{(j)}, \sum_{j=1}^{n} S h_{j}(\mu, N) f_{(j)}\right]\right),
\end{aligned}
$$

where (') is a permutation on $N$ such that $\tilde{v}_{(j)}$ being the $j$ th largest value of $\tilde{v}_{i}=\left(\mu_{i}, v_{i}\right)$.

\subsection{Models for the optimal weight vectors}

To calculate the comprehensive Atanassov's intervalvalued intuitionistic fuzzy prospect gain and loss values, it uses the weight vectors on the element set and on the ordered set. If the weight information is not exactly known, then it needs first to obtain the weight vectors on them.

Since all alternatives are no-inferior, and the optimal weight vector makes the comprehensive values of alternatives the bigger the better. Consider the multi-attribute decision making problem given in section 3.1 , if the weight vector on the attribute set $C$ is not exactly known, then we build the following model to obtain the optimal weight vector $\omega$.

$$
\begin{aligned}
& \max \sum_{i=1}^{m} \sum_{j=1}^{n} \omega_{j}
\end{aligned}
$$

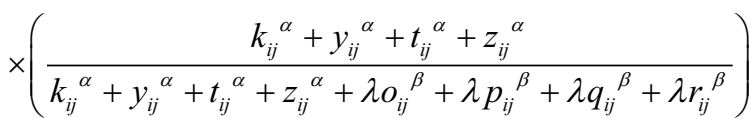

$$
\begin{aligned}
& \text { s.t. }\left\{\begin{array}{l}
\sum_{j=1}^{n} \omega_{j}=1 \\
\omega_{j} \in W_{c_{j}}, j=1,2, \ldots, n
\end{array},\right.
\end{aligned}
$$

where $W_{c_{j}}$ is the known weight information of the attribute $c_{j}, j=1,2, \ldots, n$.

Similarly, if the weight vector on the ordered set $N$ is not exactly known, then we establish the following model to obtain the optimal weight vector $w$.

$$
\begin{gathered}
\max \sum_{i=1}^{m} \sum_{j=1}^{n} w_{j}\left(k_{i(j)}{ }^{\alpha}+y_{i(j)}{ }^{\alpha}+t_{i(j)}{ }^{\alpha}+z_{i(j)}{ }^{\alpha}-\right. \\
\left.\lambda\left(o_{i(j)}{ }^{\beta}+p_{i(j)}{ }^{\beta}+q_{i(j)}{ }^{\beta}+r_{i(j)}{ }^{\beta}\right)\right) \\
\text { s.t. }\left\{\begin{array}{c}
\sum_{j=1}^{n} w_{j}=1 \\
w_{j} \in W_{j}, j=1,2, \ldots, n
\end{array}\right.
\end{gathered}
$$

where $W_{j}$ is the known weight information of the $j$ th position $j=1,2, \ldots, n$, and $(\cdot)$ is a permutation on $N$ for each $i=1,2, \ldots, m$ such that $\tilde{\tau}_{i(j)}$ and $\tilde{\pi}_{i(j)}$ being the $j$ th largest values of $\tilde{\tau}_{i j}$ and $\tilde{\pi}_{i j}$.

To consider the interactions between elements in a set, by the Shapley function the following model for the optimal fuzzy measure $\mu$ on the attribute set $C$ is constructed.

$$
\begin{aligned}
& \max \sum_{i=1}^{m} \sum_{j=1}^{n} S h_{c_{j}}(v, C)
\end{aligned}
$$

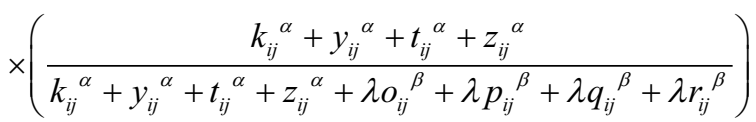

$$
\begin{aligned}
& \text { s.t. }\left\{\begin{array}{l}
v(C)=1 \\
v(S) \leq v(T) \quad \forall S, T \subseteq C \text { s.t. } S \subseteq T . \\
v\left(c_{j}\right) \in W_{c_{j}}, v\left(c_{j}\right) \geq 0, j=1,2, \ldots, n
\end{array} .\right.
\end{aligned}
$$

Similarly, model for the optimal fuzzy measure $v$ on the ordered set $N$ is constructed as follows:

$$
\begin{aligned}
& \max \sum_{i=1}^{m} \sum_{j=1}^{n} S h_{j}(\mu, N)\left({k_{i(j)}}^{\alpha}+y_{i(j)}{ }^{\alpha}+t_{i(j)}{ }^{\alpha}+z_{i(j)}{ }^{\alpha}\right. \\
& \left.-\lambda\left(o_{i(j)}{ }^{\beta}+p_{i(j)}{ }^{\beta}+q_{i(j)}{ }^{\beta}+r_{i(j)}{ }^{\beta}\right)\right) \\
& \text { s.t. }\left\{\begin{array}{l}
\mu(N)=1 \\
\mu(S) \leq \mu(T) \quad \forall S, T \subseteq N \text { s.t.S } \subseteq T . \\
\mu(j) \in W_{j}, \mu(j) \geq 0, j=1,2, \ldots, n
\end{array} .\right.
\end{aligned}
$$




\subsection{The decision procedure}

Based on Atanassov's interval-valued intuitionistic fuzzy prospect gain and loss matrices, this section develops a method to multi-attribute decision making with Atanassov's interval-valued intuitionistic fuzzy information based on prospect theory. It contains the following main steps

Step 1: Assume that $A=\left(\tilde{v}_{i j}\right)_{m \times n}$ is the IVIFN decision matrix given by the experts, where $\tilde{v}_{i j}=\left(\left[a_{i j}, b_{i j}\right],\left[c_{i j}, d_{i j}\right]\right)$ is the IVIFN for $a_{i}$ with respect to $c_{j}$.

Step 2: If all the attributes $c_{j}(j=1,2, \ldots, n)$ are benefits, then turn to Step 3, otherwise, it needs to transform the IVIFV matrix $\tilde{A}=\left(\tilde{v}_{i j}\right)_{m \times n}$ into

$$
\tilde{R}=\left(\begin{array}{cccc}
\tilde{\gamma}_{11} & \tilde{\gamma}_{12} & \ldots & \tilde{\gamma}_{1 n} \\
\tilde{\gamma}_{21} & \tilde{\gamma}_{22} & \ldots & \tilde{\gamma}_{2 n} \\
& \ldots & \ldots & \\
\tilde{\gamma}_{m 1} & \tilde{\gamma}_{m 2} & \ldots & \tilde{\gamma}_{m n}
\end{array}\right),
$$

where $\tilde{\gamma}_{i j}=\left\{\begin{array}{l}\tilde{v}_{i j} \text { for benefit attribute } c_{j} \\ \left(\tilde{v}_{i j}\right)^{C} \text { for cost attribute } c_{j}\end{array} \quad(i=1\right.$, $2, \ldots, m ; j=1,2, \ldots, n), \quad$ and $\tilde{\gamma}_{i j}=$ $\left(\left[c_{i j}, d_{i j}\right],\left[a_{i j}, b_{i j}\right]\right)(i=1,2, \ldots, m ; j=1,2, \ldots, n)$ (see [41]).

Step 3: With respect to the IVIFN reference vector $\tilde{S}=\left(\tilde{s}_{1}, \tilde{s}_{2}, \ldots, \tilde{s}_{n}\right)$, calculate the gain IVIFN decision matrix $\tilde{G}=\left(\tilde{\theta}_{i j}\right)_{m \times n}$ and the loss IVIFN decision matrix $\tilde{L}=\left(\tilde{\zeta}_{i j}\right)_{m \times n}$.

Step 4: Calculate Atanassov's interval-valued intuitionistic fuzzy prospect gain and loss matrices

$$
\begin{aligned}
& \vee G=\left(\tilde{\tau}_{i j}\right)_{m \times n}=\left(\left(\left[u_{i j l}{ }^{G}, u_{i j r}{ }^{G}\right],\left[v_{i j l}{ }^{G}, v_{i j r}{ }^{G}\right]\right)\right)_{m \times n} \\
& \text { and } \\
& V L=\left(\tilde{\pi}_{i j}\right)_{m \times n}=\left(\left(\left[u_{i j l}{ }^{L}, u_{i j r}{ }^{L}\right],\left[v_{i j l}{ }^{L}, v_{i j r}{ }^{L}\right]\right)\right)_{m \times n} .
\end{aligned}
$$

Step 5: Use models (4) and (5) to get the weight vectors on the attribute set and on the ordered set.

Step 6: Use the IVIHWA operator to compute the comprehensive Atanassov's interval-valued intuitionistic fuzzy prospect gain value $\tilde{\tau}_{i}=\operatorname{IVIHWA}_{w, \omega}\left(\tilde{\tau}_{i 1}, \tilde{\tau}_{i 2}, \ldots, \tilde{\tau}_{i n}\right)$

$$
\begin{array}{r}
=\left(\left[\frac{\sum_{j=1}^{n} w_{j} \omega_{(j)} u_{i(j) l}{ }^{G}}{\sum_{j=1}^{n} w_{j} \omega_{(j)}}, \frac{\sum_{j=1}^{n} w_{j} \omega_{(j)} u_{i(j) r}}{\sum_{j=1}^{n} w_{j} \omega_{(j)}}\right],\right. \\
\left.\left[\frac{\sum_{j=1}^{n} w_{j} \omega_{(j)} v_{i(j) l}{ }^{G}}{\sum_{j=1}^{n} w_{j} \omega_{(j)}}, \frac{\sum_{j=1}^{n} w_{j} \omega_{(j)} v_{i(j) r}}{\sum_{j=1}^{n} w_{j} \omega_{(j)}}\right]\right),
\end{array}
$$

and the comprehensive Atanassov's interval-valued intuitionistic fuzzy prospect loss value

$$
\begin{aligned}
& \tilde{\pi}_{i}=\operatorname{IVIHWA}_{w, \omega}\left(\tilde{\pi}_{i 1}, \tilde{\pi}_{i 2}, \ldots, \tilde{\pi}_{i n}\right) \\
& =\left(\left[\frac{\sum_{j=1}^{n} w_{j} \omega_{(j)} u_{i(j) l} L}{\sum_{j=1}^{n} w_{j} \omega_{(j)}}, \frac{\sum_{j=1}^{n} w_{j} \omega_{(j)} u_{i(j) r}{ }^{L}}{\sum_{j=1}^{n} w_{j} \omega_{(j)}}\right],\right. \\
& \left.\quad\left[\frac{\sum_{j=1}^{n} w_{j} \omega_{(j)} v_{i(j) l} L}{\sum_{j=1}^{n} w_{j} \omega_{(j)}}, \frac{\sum_{j=1}^{n} w_{j} \omega_{(j)} v_{i(j) r} L}{\sum_{j=1}^{n} w_{j} \omega_{(j)}}\right]\right), \\
& i=1,2, \ldots, m .
\end{aligned}
$$

Step 7: Calculate the absolute index $A I\left(\tilde{\tau}_{i}, \tilde{\pi}_{i}\right)$ and the relative index $R I\left(\tilde{\tau}_{i}, \tilde{\pi}_{i}\right), i=1,2, \ldots, m$. Then, rank the alternatives according to the absolute and relative indices.

Step 8: Select the best choice(s).

Remark 3.3 According to the procedure for the multi-attribute decision making with Atanassov's interval-valued intuitionistic fuzzy information based on prospect theory, one can easily drive a decision method to multi-attribute decision making with Atanassov's intuitionistic fuzzy information based on prospect theory. Furthermore, if we further consider the interactive characteristics between elements, by models (6) and (7) we can obtain the optimal fuzzy measures on the attribute set and on the ordered set. Then, the comprehensive attribute values of the alternatives can be obtained using the IVIHSWA operator.

\section{Two illustrative examples}

Example 4.1 According to the suppliers' core competencies, a manufacturing company intends to select the best one (adapted from Ref. [46]). There are four suppliers $\left\{a_{1}, a_{2}, a_{3}, a_{4}\right\}$ are evaluated according to the four factors: (1) $c_{1}$ is the technology innovation ability; (2) $c_{2}$ is the flow control ability; (3) $c_{3}$ is the management ability; (4) $c_{4}$ is the service level.

The four possible alternatives $a_{i}(i=1,2,3,4)$ are to be evaluated utilizing the Atanassov's intuitionistic fuzzy information by a panel of experts. The IFN decision matrix is listed as follows: 


$$
\tilde{A}^{\prime}=\left(\begin{array}{llll}
(0.5,0.3) & (0.5,0.4) & (0.7,0.2) & (0.3,0.1) \\
(0.4,0.3) & (0.3,0.4) & (0.9,0.1) & (0.5,0.2) \\
(0.4,0.1) & (0.5,0.3) & (0.5,0.4) & (0.6,0.2) \\
(0.6,0.2) & (0.2,0.5) & (0.4,0.2) & (0.7,0.1)
\end{array}\right) .
$$

With respect to these four factors, the IFN reference vector is given by

$$
\tilde{S}=((0.5,0.2),(0.4,0.3),(0.6,0.1),(0.6,0.2)) .
$$

For example, the experts think that a good supplier's the level of technology innovation with $50 \%$, and its insufficiency with respect to the level of technology innovation with $20 \%$. Suppose the attribute weights are given by $\omega_{1}=0.3, \omega_{2}=2, \omega_{3}=0.2$, and $\omega_{4}=0.3$, and the ordered position weights are defined by $w_{1}=0.2, w_{2}=0.3, w_{3}=0.3$, and $w_{4}=0.2$. To effectively solve this problem, the following steps are contained.

Step 1: Since all attributes are benefits, there is no need to normalize IFN decision matrix. From the IFN reference vector, the gain IFN decision matrix is obtained by

$$
G^{\prime}=\left(\begin{array}{cccc}
(0,0) & (0.1,0.1) & (0.1,0) & (0,0) \\
(0,0) & (0,0) & (0.3,0) & (0,0) \\
(0,0.1) & (0.1,0) & (0,0) & (0,0) \\
(0.1,0) & (0,0) & (0,0) & (0.1,0.1)
\end{array}\right),
$$

and the loss IFN decision matrix is got by

$$
E^{\prime}=\left(\begin{array}{cccc}
(0,0.1) & (0,0.1) & (0,0.1) & (0.3,0) \\
(0.1,0.1) & (0.1,0.1) & (0,0) & (0.1,0) \\
(0.1,0) & (0,0) & (0.1,0.3) & (0,0) \\
(0,0) & (0.2,0.2) & (0.2,0.1) & (0,0)
\end{array}\right) .
$$

Step 2: Let $\alpha=\beta=0.88$, and $\lambda=2.25$, according to Kahneman and Tversky [23], it gets the following Atanassov's intuitionistic fuzzy prospect gain and loss matrices

$$
\begin{aligned}
\nabla G^{\prime} & =\left(\begin{array}{cccc}
(0,0) & (0.132,0.132) & (0.132,0) & (0,0) \\
(0,0) & (0,0) & (0.347,0) & (0,0) \\
(0,0.132) & (0.132,0) & (0,0) & (0,0) \\
(0.132,0) & (0,0) & (0,0) & (0.132,0.132)
\end{array}\right), \\
V L^{\prime} & =\left(\begin{array}{cccc}
(0,-0.3) & (0,-0.3) & (0,-0.3) & (-0.78,0) \\
(-0.3,-0.3) & (-0.3,-0.3) & (0,0) & (-0.3,0) \\
(-0.3,0) & (0,0) & (-0.78,-0.78) & (0,0) \\
(0,0) & (-0.546,-0.546) & (-0.546,-0.3) & (0,0)
\end{array}\right) .
\end{aligned}
$$

Step 3: Use the IHWA operator to calculate the comprehensive Atanassov's intuitionistic fuzzy prospect gain value, take $\tilde{\tau}_{1}$, for example,

$$
\begin{aligned}
\tilde{\tau}_{1=}^{\prime}= & \operatorname{IHWA}_{w, \omega}\left(\tilde{\tau}_{11}^{\prime}, \tilde{\tau}_{12}^{\prime}, \tilde{\tau}_{13}^{\prime}, \tilde{\tau}_{14}^{\prime}\right) \\
= & \left(\frac{0.2 \times 0.2 \times 0.132+0.2 \times 0.3 \times 0.132}{0.2 \times 0.2+0.2 \times 0.3+0.3 \times 0.3+0.2 \times 0.3},\right. \\
& \left.\frac{0.2 \times 0.2 \times 0.132}{0.2 \times 0.2+0.2 \times 0.3+0.3 \times 0.3+0.2 \times 0.3}\right) \\
= & (0.053,0.021) .
\end{aligned}
$$

Similar to the calculation of the comprehensive Atanassov's intuitionistic fuzzy prospect gain value $\tilde{\tau}_{1}^{\prime}$, it has

$$
\begin{aligned}
& \tilde{\tau}_{2}^{\prime}=(0.021,0), \tilde{\tau}_{3}^{\prime}=(0.033,0.033), \\
& \tilde{\tau}_{4}^{\prime}=(0.079,0.032) .
\end{aligned}
$$

Furthermore, the comprehensive Atanassov's intuitionistic fuzzy prospect loss values are obtained by

$$
\begin{gathered}
\tilde{\pi}_{1}^{\prime}=(-0.195,-0.222), \tilde{\pi}_{2}^{\prime}=(-0.251,-0.148), \\
\tilde{\pi}_{3}^{\prime}=(-0.148,-0.12), \tilde{\pi}_{4}^{\prime}=(-0.218,-0.159) .
\end{gathered}
$$

Step 4: According to the comprehensive Atanassov's intuitionistic fuzzy prospect gain and loss values, it has

$$
\begin{aligned}
& A I\left(\tilde{\tau}_{1}^{\prime}, \tilde{\pi}_{1}^{\prime}\right)=-0.345, A I\left(\tilde{\tau}_{2}^{\prime}, \tilde{\pi}_{2}^{\prime}\right)=-0.378, \\
& A I\left(\tilde{\tau}_{3}^{\prime}, \tilde{\pi}_{3}^{\prime}\right)=-0.202, A I\left(\tilde{\tau}_{4}^{\prime}, \tilde{\pi}_{4}^{\prime}\right)=-0.266 .
\end{aligned}
$$

From $A I\left(\tilde{\tau}_{i}^{\prime}, \tilde{\pi}_{i}^{\prime}\right), i=1,2,3,4$, it gets $a_{2} \prec a_{1} \prec a_{4} \prec a_{3}$. Namely, $a_{3}$ is the best supplier.

Example 4.2 Consider an investment firm, which wants to invest some money in some firm for the best profits (adapted from Ref. [47]). There are four alternatives: (1) a car firm $a_{1}$; (2) a food firm $a_{2}$; (3) a computer firm $a_{3}$; (4) an arms firm $a_{4}$. These four firms $\left\{a_{1}, a_{2}, a_{3}, a_{4}\right\}$ are evaluated according to three attributes: (1) $c_{1}$ is influence on risk; (2) $c_{2}$ is the ability of growth; (3) $c_{3}$ is influence on environment. The evaluations of the four possible alternatives given by the experts are shown as in the following IVIFN decision matrix:

$$
\tilde{A}=\left(\begin{array}{lll}
([0.3,0.4],[0.4,0.5]) & ([0.4,0.6],[0.2,0.4]) & ([0.2,0.3],[0.5,0.6]) \\
([0.2,0.3],[0.6,0.7]) & ([0.6,0.7],[0.2,0.3]) & ([0.1,0.2],[0.4,0.7]) \\
([0.3,0.4],[0.3,0.6]) & ([0.5,0.6],[0.3,0.4]) & ([0.2,0.3],[0.5,0.6]) \\
([0.1,0.2],[0.7,0.8]) & ([0.6,0.7],[0.1,0.3]) & ([0.1,0.2],[0.3,0.4])
\end{array}\right) .
$$

Each element of this matrix represents reflects the experts' belief that each alternative are an excellent investment as well as not an excellent choice. The weight vector on attribute set is not exactly determined, and the experts only give their ranges, denoted by $W_{c_{1}}=[0.25,0.3], \quad W_{c_{2}}=[0.35,0.4]$ and $W_{c_{3}}=[0.25,0.4]$.

Furthermore, to decrease the influence of the extreme value, the weight vector on the ordered set $N$ $=\{1,2,3\}$ are defined by $W_{1}=[0.2,0.4]$, $W_{2}=[0.3,0.5]$ and $W_{3}=[0.2,0.4]$. The IVIFN reference vector with respect to each attribute is given as follows:

$$
\begin{gathered}
\tilde{S}=(([0.2,0.4],[0.5,0.6]),([0.4,0.5],[0.3,0.4]), \\
([0.2,0.3],[0.4,0.5])) .
\end{gathered}
$$

Take the first element in $\tilde{S}$ for example; the experts think that a good investee firm's risk tolerance with a 
margin of $50 \%-60 \%$, and its ability cannot afford risk with between $20 \%$ and $40 \%$. To obtain the best investment firm(s), the following decision procedure is followed.

Step 1: Since the attributes $c_{1}$ and $c_{3}$ are cost, we first transform the IVIFN decision matrix into

$$
\tilde{R}=\left(\begin{array}{lll}
([0.4,0.5],[0.3,0.4]) & ([0.4,0.6],[0.2,0.4]) & ([0.5,0.6],[0.2,0.3]) \\
([0.6,0.7],[0.2,0.3]) & ([0.6,0.7],[0.2,0.3]) & ([0.4,0.7],[0.1,0.2]) \\
([0.3,0.6],[0.3,0.4]) & ([0.5,0.6],[0.3,0.4]) & ([0.5,0.6],[0.2,0.3]) \\
([0.7,0.8],[0.1,0.2]) & ([0.6,0.7],[0.1,0.3]) & ([0.3,0.4],[0.1,0.2])
\end{array}\right) .
$$

Furthermore, the IVIFN reference vector is modified as

$$
\begin{aligned}
\tilde{S}^{\prime}= & (([0.5,0.6],[0.2,0.4]),([0.4,0.5],[0.3,0.4]), \\
& ([0.4,0.5],[0.2,0.3])) .
\end{aligned}
$$

Step 2: With respect to $\tilde{S}^{\prime}$, interval-valued Atanassov's intuitionistic fuzzy prospect gain matrix is obtained by

$$
G=\left(\begin{array}{ccc}
([0,0],[0,0]) & ([0,0.1],[0,0.1]) & ([0.1,0.1],[0,0]) \\
([0.1,0.1],[0,0.1]) & ([0.2,0.2],[0.1,0.1]) & ([0.2,0.4],[0.1,0.1]) \\
([0,0],[0,0]) & ([0.1,0.1],[0,0]) & ([0.1,0.1],[0,0]) \\
([0.2,0.2],[0.1,0.2]) & ([0.2,0.2],[0.1,0.2]) & ([0,0],[0.1,0.1])
\end{array}\right) .
$$

Furthermore, Atanassov's interval-valued intuitionistic fuzzy prospect loss matrix is got by

$$
Z=\left(\begin{array}{ccc}
([0.1,0.1],[0,0.1]) & ([0,0],[0,0]) & ([0,0],[0,0]) \\
([0,0],[0,0]) & ([0,0],[0,0]) & ([0,0],[0,0]) \\
([0,0.2],[0,0.1]) & ([0,0],[0,0]) & ([0,0],[0,0]) \\
([0,0],[0,0]) & ([0,0],[0,0]) & ([0.1,0.1],[0,0])
\end{array}\right) .
$$

Step 3: Let $\alpha=\beta=0.88$, and $\lambda=2.25$, according to Kahneman and Tversky [23], Atanassov's intervalvalued intuitionistic fuzzy prospect gain matrix is obtained

$$
\nabla G=\left(\begin{array}{cc}
([0,0],[0,0]) & ([0,0.132],[0,0.132]) \\
([0.132,0.132],[0,0.132]) & ([0.243,0.243],[0.132,0.132]) \\
([0,0],[0,0]) & ([0.132,0.132],[0,0]) \\
([0.243,0.243],[0.132,0.243]) & ([0.243,0.243],[0.132,0.243]) \\
([0.132,0.132],[0,0]) \\
([0.243,0.446],[0.132,0.132]) \\
([0.132,0.132],[0,0]) \\
([0,0],[0.132,0.132])
\end{array}\right),
$$

and Atanassov's interval-valued intuitionistic fuzzy prospect loss matrix is got

$$
\mathscr{V} L=\left(\begin{array}{ccc}
([-0.3,-0.3],[-0.3,0]) & ([0,0],[0,0]) & ([0,0],[0,0]) \\
([0,0],[0,0]) & ([0,0],[0,0]) & ([0,0],[0,0]) \\
([-0.546,0],[-0.3,0]) & ([0,0],[0,0]) & ([0,0],[0,0]) \\
([0,0],[0,0]) & ([0,0],[0,0]) & ([-0.3,-0.3],[0,0])
\end{array}\right) .
$$

Step 4: From model (4), it drives the following programming for the optimal weight vector on the attribute set $C=\left\{c_{1}, c_{2}, c_{3}\right\}$.

$$
\begin{gathered}
\max 2 \omega_{1}+4 \omega_{2}+3.306 \omega_{3} \\
\text { s.t. }\left\{\begin{array}{l}
\omega_{1}+\omega_{2}+\omega_{3}=1 \\
\omega_{1} \in[0.25,0.3], \omega_{2} \in[0.35,0.4] . \\
\omega_{3} \in[0.25,0.4]
\end{array}\right.
\end{gathered}
$$

Solve the above linear programming, it has $\omega_{1}=0.25$, $\omega_{2}=0.4$, and $\omega_{3}=0.35$.

From model (5), it drives the following programming for the optimal weight vector on the ordered set $N=$ $\{1,2,3\}$.

$$
\begin{aligned}
& \max 2.342 w_{1}+2.139 w_{2}-1.686 w_{3} \\
& \text { s.t. }\left\{\begin{array}{l}
w_{1}+w_{2}+w_{3}=1 \\
w_{1} \in[0.2,0.4], w_{2} \in[0.3,0.5] . \\
w_{3} \in[0.2,0.4]
\end{array}\right.
\end{aligned}
$$

Solve the above linear programming, it has $w_{1}=0.4$, $w_{2}=0.4$, and $w_{3}=0.2$.

Step 5: According to the IVIHWA operator, the comprehensive Atanassov's interval-valued intuitionistic fuzzy prospect gain value of the alternative $a_{1}$ is obtained as follows:

$$
\begin{aligned}
\tilde{\tau}_{1}= & \operatorname{IVIHWA}_{w, \omega}\left(\tilde{\tau}_{11}, \tilde{\tau}_{12}, \tilde{\tau}_{13}\right) \\
= & \left(\left[\frac{0.4 \times 0.35 \times 0.132}{0.4 \times 0.4+0.4 \times 0.35+0.2 \times 0.25},\right.\right. \\
& \left.\frac{0.4 \times 0.4 \times 0.132+0.4 \times 0.35 \times 0.132}{0.4 \times 0.4+0.4 \times 0.35+0.2 \times 0.25}\right], \\
= & {\left.\left[0, \frac{0.4 \times 0.4 \times 0.132}{0.4 \times 0.4+0.4 \times 0.35+0.2 \times 0.25}\right]\right) } \\
= & ([0.053,0.113],[0,0.06]) .
\end{aligned}
$$

Similar to the calculation of $\tilde{\tau}_{1}$, it has

$$
\begin{aligned}
& \tilde{\tau}_{2}=([0.227,0.308],[0.113,0.132]), \\
& \tilde{\tau}_{3}=([0.113,0.113],[0,0]), \\
& \tilde{\tau}_{4}=([0.191,0.191],[0.132,0.219]) .
\end{aligned}
$$

Similar to the calculation of the comprehensive Atanassov's interval-valued intuitionistic fuzzy prospect gain values, the comprehensive Atanassov's interval-valued intuitionistic fuzzy prospect loss values are obtained by

$$
\begin{aligned}
\tilde{\pi}_{1} & =([-0.043,-0.043],[-0.043,0]), \\
\tilde{\pi}_{2} & =([0,0],[0,0]), \\
\tilde{\pi}_{3} & =([-0.078,0],[-0.043,0]), \\
\tilde{\pi}_{4} & =([-0.064,-0.064],[0,0]) .
\end{aligned}
$$

Step 6: According to the comprehensive Atanassov's interval-valued intuitionistic fuzzy prospect gain and loss values, it derives 


$$
\begin{aligned}
& A I\left(\tilde{\tau}_{1}, \tilde{\pi}_{1}\right)=0.098, A I\left(\tilde{\tau}_{2}, \tilde{\pi}_{2}\right)=0.781, \\
& A I\left(\tilde{\tau}_{3}, \tilde{\pi}_{3}\right)=0.105, A I\left(\tilde{\tau}_{4}, \tilde{\pi}_{4}\right)=0.607 .
\end{aligned}
$$

By $A I\left(\tilde{\tau}_{i}, \tilde{\pi}_{i}\right), i=1,2,3,4$, it gets $a_{1} \prec a_{3} \prec a_{4} \prec a_{2}$. Namely, the best choice is $a_{2}$ (food firm).

In this example, if we further consider the interactions between attributes and between ordered positions, then the main procedure is listed as follows:

Step 1: Base on Atanassov's interval-valued intuitionistic fuzzy prospect gain and loss matrices $V G$ and $V L$, it drives the following programming for the optimal fuzzy measure $v$ on the attribute set $C=$ $\left\{c_{1}, c_{2}, c_{3}\right\}$.

$$
\begin{aligned}
& \max -0.551\left(v\left(c_{1}\right)-v\left(c_{2}, c_{3}\right)\right)+0.449\left(v\left(c_{2}\right)-v\left(c_{1}, c_{3}\right)\right) \\
&+0.102\left(v\left(c_{3}\right)-v\left(c_{1}, c_{2}\right)\right)+3.102 \\
& \text { s.t. }\left\{\begin{array}{l}
v\left(c_{1}\right) \in[0.25,0.3], v\left(c_{2}\right) \in[0.35,0.4] \\
v\left(c_{3}\right) \in[0.25,0.4] \\
v(S) \leq v(T) \quad \forall S, T \subseteq\left\{c_{1}, c_{2}, c_{3}\right\} \text { s.t. } S \subseteq T
\end{array}\right.
\end{aligned}
$$

Solve the above linear programming, it gives

$$
\begin{aligned}
& v\left(c_{1}\right)=v\left(c_{3}\right)=v\left(c_{1}, c_{3}\right)=0.25, v\left(c_{2}\right)=v\left(c_{1}, c_{2}\right)=0.4, \\
& v\left(c_{2}, c_{3}\right)=v\left(c_{1}, c_{2}, c_{3}\right)=1 .
\end{aligned}
$$

By model (7), it drives the following programming for the optimal fuzzy measure $\mu$ on the ordered set $N$ $=\{1,2,3\}$.

$$
\begin{aligned}
& \max -0.705(\mu(1)-\mu(2,3))+0.604(\mu(2)-\mu(1,3)) \\
&- 1.309(\mu(3)-\mu(1,2))+0.932 \\
& \text { s.t. }\left\{\begin{array}{l}
\mu(1) \in[0.2,0.4], \mu(2) \in[0.3,0.5] \\
\mu(3) \in[0.2,0.4] \\
\mu(S) \leq \mu(T) \quad \forall S, T \subseteq\{1,2,3\} \text { s.t. } S \subseteq T
\end{array}\right.
\end{aligned}
$$

Solve the above linear programming, it gives

$$
\begin{aligned}
& \mu(1)=\mu(1,3)=0.4, \mu(2)=\mu(2,3)=0.3, \mu(3)=0.2, \\
& \mu(1,2)=\mu(1,2,3)=1 .
\end{aligned}
$$

From the fuzzy measure $v$, the attributes' Shapley values are

$$
\begin{aligned}
& S h_{c_{1}}(v, C)=0.0833, S h_{c_{2}}(v, C)=0.5333, \\
& S h_{c_{3}}(v, C)=0.3833 .
\end{aligned}
$$

Furthermore, by the fuzzy measure $\mu$ the ordered positions' Shapley values are

$$
\begin{aligned}
& S h_{1}(\mu, N)=0.517, S_{2}(\mu, N)=0.417, \\
& S h_{3}(\mu, N)=0.067 .
\end{aligned}
$$

Step 2: Using the IVIHSWA operator, the comprehensive Atanassov's interval-valued intuitionistic fuzzy prospect gain values are obtained as follows:

$$
\begin{aligned}
& \tilde{\tau}_{1}^{\prime}=([0.061,0.13],[0,0.069]), \\
& \tilde{\tau}_{2}^{\prime}=([0.242,0.336],[0.13,0.132]), \\
& \tilde{\tau}_{3}^{\prime}=([0.13,0.13],[0,0]), \\
& \tilde{\tau}_{4}^{\prime}=([0.222,0.222],[0.132,0.233]) .
\end{aligned}
$$

Furthermore, by the IVIHSWA operator the comprehensive Atanassov's interval-valued intuitionistic fuzzy prospect loss values are obtained as follows:

$$
\begin{aligned}
\tilde{\pi}_{1}^{\prime} & =([-0.004,-0.004],[-0.004,0]), \\
\tilde{\pi}_{2}^{\prime} & =([0,0],[0,0]), \\
\tilde{\pi}_{3}^{\prime} & =([-0.007,0],[-0.004,0]), \\
\tilde{\pi}_{4} & =([-0.026,-0.026],[0,0]) .
\end{aligned}
$$

Step 3: According to the comprehensive Atanassov's interval-valued intuitionistic fuzzy prospect gain and loss values, it gives

$$
\begin{gathered}
A I\left(\tilde{\tau}_{1}^{\prime}, \tilde{\pi}_{1}^{\prime}\right)=0.249, A I\left(\tilde{\tau}_{2}^{\prime}, \tilde{\pi}_{2}^{\prime}\right)=0.84, \\
A I\left(\tilde{\tau}_{3}^{\prime}, \tilde{\pi}_{3}^{\prime}\right)=0.25, A I\left(\tilde{\tau}_{4}, \tilde{\pi}_{4}\right)=0.756 .
\end{gathered}
$$

By $A I\left(\tilde{\tau}_{i}^{\prime}, \tilde{\pi}_{i}^{\prime}\right), i=1,2,3,4$, it gives $a_{1} \prec a_{3} \prec a_{4} \prec a_{2}$. Namely, the best choice is $a_{2}$ (food firm).

It is interesting that the same ranking order is obtained, and the best choice is $a_{2}$ in both cases. If we do not consider the IVIFN reference vector and directly calculate the comprehensive Atanassov's interval-valued intuitionistic fuzzy values using some aggregation operators, the ranking order is obtained as shown in Table 1.

From Table 1, one can see that all ranking orders obtained from the aggregation operators based on additive measures are different to that derived the above ranking order. However, the same ranking order is obtained from the aggregation operators based on fuzzy measures.

Remark 4.1 As we know, the ranking order may be varied with the ranking methods. Thus, it is necessary to further consider the ranking approaches for Atanassov's interval-valued intuitionistic fuzzy prospect gain and loss values. It is worth pointing out that the ranking method defined in this paper can be seen as a special case of the generated admissible order for intervals $[49,50]$. Following the works of Bustince et al. [49, 50], we introduce the following generated admissible order between Atanassov's interval-valued intuitionistic fuzzy prospect gain and loss values using two continuous functions. 
Table 1 The ranking order with respect to the different aggregation operators

\begin{tabular}{|c|c|c|c|c|c|}
\hline & \multicolumn{4}{|c|}{ The comprehensive Atanassov's interval-valued intuitionistic fuzzy values } & \multirow{2}{*}{ The ranking order } \\
\hline & $\tilde{v}_{1}$ & $\tilde{v}_{2}$ & $\tilde{v}_{3}$ & $\tilde{v}_{4}$ & \\
\hline The IIWAA operator [13] & $([0.44,0.58],[0.22,0.36])$ & $([0.54,0.7],[0.16,0.26])$ & $([0.46,0.6],[0.26,0.36])$ & $([0.55,0.65],[0.1,0.24])$ & $a_{1} \prec a_{3} \prec a_{2} \prec a_{4}$ \\
\hline The IIOWA operator [13] & $([0.44,0.58],[0.22,0.36])$ & $([0.57,0.7],[0.17,0.28])$ & $([0.46,0.6],[0.26,0.36])$ & $([0.6,0.71],[0.1,0.24])$ & $a_{1} \prec a_{3} \prec a_{2} \prec a_{4}$ \\
\hline The IIHA operator [13] & $([0.46,0.6],[0.2,0.34])$ & $([0.55,0.72],[0.14,0.24])$ & $([0.49,0.62],[0.24,0.34])$ & $([0.58,0.69],[0.1,0.25])$ & $a_{1} \prec a_{3} \prec a_{2} \prec a_{4}$ \\
\hline The IIFWGA operator [12] & $([0.43,0.57],[0.23,0.37])$ & $([0.52,0.7],[0.17,0.27])$ & $([0.44,0.6],[0.27,0.37])$ & $([0.49,0.6],[0.1,0.24])$ & $a_{3} \prec a_{1} \prec a_{4} \prec a_{2}$ \\
\hline The IIFOWGA operator [12] & $([0.44,0.58],[0.22,0.36])$ & $([0.55,0.7],[0.18,0.28])$ & $([0.45,0.6],[0.26,0.36])$ & $([0.56,0.66],[0.1,0.24])$ & $a_{3} \prec a_{1} \prec a_{2} \prec a_{4}$ \\
\hline The IIFWHGA operator [12] & $([0.46,0.58],[0.22,0.35])$ & $([0.52,0.71],[0.15,0.25])$ & $([0.44,0.61],[0.25,0.35])$ & $([0.55,0.65],[0.1,0.25])$ & $a_{3} \prec a_{1} \prec a_{2} \prec a_{4}$ \\
\hline The IVIFCA operator [14] & $([0.43,0.6],[0.2,0.37])$ & $([0.56,0.7],[0.17,0.27])$ & $([0.5,0.6],[0.27,0.37])$ & $([0.48,0.59],[0.1,0.21])$ & $a_{1} \prec a_{3} \prec a_{4} \prec a_{2}$ \\
\hline The IVIFCG operator [14] & $([0.42,0.6],[0.2,0.38])$ & $([0.54,0.7],[0.18,0.28])$ & $([05,0.6],[0.28,0.38])$ & $([0.41,0.52],[0.1,0.22])$ & $a_{1} \prec a_{3} \prec a_{4} \prec a_{2}$ \\
\hline The IVIFHSA operator [42] & $([0.44,0.6],[0.2,0.36])$ & $([0.54,0.7],[0.16,0.26])$ & $([0.5,0.6],[0.26,0.36])$ & $([0.51,0.62],[0.1,0.26])$ & $a_{1} \prec a_{3} \prec a_{4} \prec a_{2}$ \\
\hline The GSAIVIFCA operator [48] & $([0.44,0.59],[0.21,0.36])$ & $([0.52,0.7],[0.16,0.26])$ & $([0.48,0.6],[0.26,0.36])$ & $([0.48,0.58],[0.1,0.25])$ & $a_{1} \prec a_{3} \prec a_{4} \prec a_{2}$ \\
\hline
\end{tabular}

Definition 4.1 Let $A$ and $B$ be two binary aggregation functions on Atanassov's interval-valued intuitionistic fuzzy prospect gain and loss values, and (IVIFVs, ${ }^{\circ}$ ) be a poset. ${ }^{\circ}$ is called an admissible order on Atanassov's IVIFVs, if

(i) ${ }^{\circ}$ is a linear order on Atanassov's IVIFVs, namely, for all Atanassov's IVIFVs $\tilde{\alpha}_{1}, \tilde{\alpha}_{2}$, either $\tilde{\alpha}_{1}{ }^{\circ} \tilde{\alpha}_{2}$ or $\tilde{\alpha}_{2}^{\circ} \tilde{\alpha}_{1}$

(ii) for all Atanassov's IVIFVs $\tilde{\alpha}_{1}, \tilde{\alpha}_{2}, \tilde{\alpha}_{1}{ }^{\circ} \tilde{\alpha}_{2}$ whenever $A\left(\tilde{v}_{1}, \tilde{\zeta}_{1}\right) \leq A\left(\tilde{v}_{2}, \tilde{\zeta}_{2}\right)$ and $B\left(\tilde{v}_{1}, \tilde{\zeta}_{1}\right) \leq B\left(\tilde{v}_{2}, \tilde{\zeta}_{2}\right)$, where $\tilde{v}_{1}, \tilde{v}_{2}$ are Atanassov's interval-valued intuitionistic fuzzy prospect gain values, and $\tilde{\zeta}_{1}, \tilde{\zeta}_{2}$ are Atanassov's interval-valued intuitionistic fuzzy prospect loss values with respect to $\tilde{\alpha}_{1}, \tilde{\alpha}_{2}$ and the given IVIFN preference points.

Definition 4.2 Let $^{\circ}$ be an admissible order on Atanassov's IVIFVs. ${ }^{\circ}$ is called a generated admissible order if there exist two continuous scalarvalued binary functions $f, g$ defined on Atanassov's interval-valued intuitionistic fuzzy prospect gain and loss values such that for all Atanassov's IVIFVs $\tilde{\alpha}_{1}$, $\tilde{\alpha}_{2} \quad, \quad \tilde{\alpha}_{1}{ }^{\circ} \quad \tilde{\alpha}_{2} \Leftrightarrow f\left(\tilde{v}_{1}, \tilde{\zeta}_{1}\right) \leq f\left(\tilde{v}_{2}, \tilde{\zeta}_{2}\right) \quad$ or $f\left(\tilde{v}_{1}, \tilde{\zeta}_{1}\right)=f\left(\tilde{v}_{2}, \tilde{\zeta}_{2}\right)$ and $g\left(\tilde{v}_{1}, \tilde{\zeta}_{1}\right) \leq g\left(\tilde{v}_{2}, \tilde{\zeta}_{2}\right)$, denoted by ${ }^{\circ}, g$, where $\tilde{v}_{1}, \tilde{v}_{2}, \tilde{\zeta}_{1}, \tilde{\zeta}_{2}$ as shown in Definition 4.1.

In Definition 4.2, one can derive that if $f(x, y)=(1-\gamma) A I(x, y)+\gamma R I(x, y)$ and $g(x, y)=(1-\eta) A I(x, y)$ $+\eta R I(x, y)$ with $\gamma \neq \eta, \gamma, \eta \in[0,1]$, then ${ }_{f, g}^{\circ}$ is the weighted mean admissible order as follows:

$$
\begin{aligned}
& \quad \text { If }(1-\gamma) A I\left(\tilde{v}_{1}, \tilde{\zeta}_{1}\right)+\gamma R I\left(\tilde{v}_{1}, \tilde{\zeta}_{1}\right)< \\
& \quad(1-\gamma) A I\left(\tilde{v}_{2}, \tilde{\zeta}_{2}\right)+\gamma R I\left(\tilde{v}_{2}, \tilde{\zeta}_{2}\right), \\
& \operatorname{then}\left(\tilde{v}_{1}, \tilde{\zeta}_{1}\right) \prec\left(\tilde{v}_{2}, \tilde{\zeta}_{2}\right) . \\
& \quad \operatorname{If}(1-\gamma) A I\left(\tilde{v}_{1}, \tilde{\zeta}_{1}\right)+\gamma R I\left(\tilde{v}_{1}, \tilde{\zeta}_{1}\right)= \\
& (1-\gamma) A I\left(\tilde{v}_{2}, \tilde{\zeta}_{2}\right)+\gamma R I\left(\tilde{v}_{2}, \tilde{\zeta}_{2}\right),
\end{aligned}
$$

then

$$
\left\{\begin{array}{l}
(1-\eta) A I\left(\tilde{v}_{1}, \tilde{\zeta}_{1}\right)+\eta R I\left(\tilde{v}_{1}, \tilde{\zeta}_{1}\right)=(1-\eta) A I\left(\tilde{v}_{2}, \tilde{\zeta}_{2}\right)+\eta R I\left(\tilde{v}_{2}, \tilde{\zeta}_{2}\right) \\
\Rightarrow\left(\tilde{v}_{1}, \tilde{\zeta}_{1}\right)=\left(\tilde{v}_{2}, \tilde{\zeta}_{2}\right) \\
(1-\eta) A I\left(\tilde{v}_{1}, \tilde{\zeta}_{1}\right)+\eta R I\left(\tilde{v}_{1}, \tilde{\zeta}_{1}\right)<(1-\eta) A I\left(\tilde{v}_{2}, \tilde{\zeta}_{2}\right)+\eta R I\left(\tilde{v}_{2}, \tilde{\zeta}_{2}\right) \\
\Rightarrow\left(\tilde{v}_{1}, \tilde{\zeta}_{1}\right) \prec\left(\tilde{v}_{2}, \tilde{\zeta}_{2}\right)
\end{array} .\right.
$$

With respect to the different values of $\gamma, \eta$, it derives the different ranking methods. For example, if $\gamma=0$, $\eta=1$, it derives the ranking method introduced in subsection 3.1.

In Example 4.2, with respect to the IVIHSWA operator, if $\gamma=1, \eta=0$, it gets

$$
\begin{aligned}
& R I\left(\tilde{\tau}_{1}^{\prime}, \tilde{\pi}_{1}^{\prime}\right)=0.956, R I\left(\tilde{\tau}_{2}^{\prime}, \tilde{\pi}_{2}^{\prime}\right)=1, \\
& R I\left(\tilde{\tau}_{3}^{\prime}, \tilde{\pi}_{3}^{\prime}\right)=0.959, \operatorname{RI}\left(\tilde{\tau}_{4}, \tilde{\pi}_{4}\right)=0.94 .
\end{aligned}
$$

Thus, the ranking order of alternatives is derived $a_{4} \prec a_{3} \prec a_{1} \prec a_{2}$, which is different to that obtained by $\gamma=0, \eta=1$. From this case, one can see the influence of the ranking method for the final ranking order. Thus, it needs the expert to decide the using ranking method before making a decision. 


\section{Conclusions}

Since prospect theory well describes the decision makers' behaviors for the gain and loss situations, it has become the most important behavioral decision making method under risk and uncertain. Based on prospect theory, this article effectively studies multi-attribute decision making with Atanassov's interval-valued intuitionistic fuzzy information. By the IVIFN preference vector on the attribute set, the IVIFN gain and loss matrices are defined. Then, the Atanassov's interval-valued intuitionistic fuzzy prospect gain and loss matrices are obtained using the value function in prospect theory. Furthermore, an order relationship is introduced. In order to get the comprehensive Atanassov's interval-valued intuitionistic fuzzy prospect gain and loss values of the alternatives, the IVIHWA and IVIHSWA operators based on additive measures and fuzzy measures are, respectively, defined. Moreover, the linear programming models are built, by which the optimal weight vectors can be obtained. As a series of development, a method to multi-attribute decision making with Atanassov's interval-valued intuitionistic fuzzy information is developed, which differs from any existing one. It is worth pointing out that the method can be directly used in the setting of Atanassov's intuitionistic fuzzy environment. Furthermore, besides the application of the developed procedure in the decision making, it can also be used in some other fields, such as industrial engineering, expert systems, neural networks, digital image processing, and uncertain systems and controls.

\section{Acknowledgements}

The authors first gratefully thank the Editor-in-Chief Prof. L. Martínez Lopez, the Associate Editor and the referee for their valuable and constructive comments which have much improved the paper. This work was supported by the State Key Program of National Natural Science of China (No. 71431006), the Funds for Creative Research Groups of China (No. 71221061), the Projects of Major International Cooperation NSFC (No. 71210003), the National Natural Science Foundation of China (Nos. 71201089, 71271217, 71201110 and 71271029), the National Science Foundation for Post-doctoral Scientists of China (2014M560655), and the Program for New Century Excellent Talents in University of China (No. NCET-12-0541).

\section{References}

[1] I. Chamodrakas, I. Leftheriotis and D. Martakos, Indepth analysis and simulation study of an innovative fuzzy approach for ranking alternatives in multiple attribute decision making problems based on TOPSIS, Appl. Soft Comput. 11(1) (2011) 900-907.

[2] Y. W. Chen and M. Larbani, Two-person zero-sum game approach for fuzzy multiple attribute decision making problems, Fuzzy Set. Syst. 157(1) (2006) 34-51.

[3] Z. P. Fan, G. F. Hu and S. H. Xiao, A method for multiple attribute decision-making with the fuzzy preference relation on alternatives, Comput. Ind. Eng. 46(2) (2004) 321-327.

[4] Z. P. Fan and B. Feng, A multiple attributes decision making method using individual and collaborative attribute data in a fuzzy environment, Inform. Sci. 179(20) (2009) 3603-3618.

[5] X. B. Gu and Q. X. Zhu, Fuzzy multi-attribute decision-making method based on eigenvector of fuzzy attribute evaluation space, Decision Supp. Syst. 41(2) (2006) 400-410.

[6] C. Kahraman and S. Çebí, A new multi-attribute decision making method: Hierarchical fuzzy axiomatic design, Expert Syst. Appl. 36(3) (2009) 4848-4861.

[7] Z. Pei, Simplification of fuzzy multiple attribute decision making in production line evaluation, Knowl-Based Syst. 47 (2013) 23-34.

[8] K. T. Atanassov and G. Gargov, Interval-valued intuitionistic fuzzy sets, Fuzzy Set. Syst. 31(2) (1989) 343-349.

[9] K. T. Atanassov, Operators over interval-valued intuitionistic fuzzy sets, Fuzzy Set. Syst. 64 (1994) 159-174.

[10] F.Y. Meng, C. Q. Tan and Q. Zhang, The induced generalized interval-valued intuitionistic fuzzy hybrid Shapley averaging operator and its application in decision making, Knowl-Based Syst. 42 (2013) 9-19.

[11] C. Q. Tan, A multi-criteria interval-valued intuitionistic fuzzy group decision making with Choquet integral-based TOPSIS, Expert Syst. Appl. 38(4) (2011) 3023-3033.

[12] G. W. Wei and X. R. Wang, Some geometric aggregation operators based on interval-valued intuitionistic fuzzy sets and their application to group decision making, in : The CIS, Harbin, China, 2007, pp.495-499.

[13] Z. S. Xu and J. Chen, Approach to group decision making based on interval-valued intuitionistic judgment matrices, Syst. Eng. - Theory and Practice 27(1) (2007) 126-133.

[14] Z. S. Xu, Choquet integrals of weighted intuitionistic fuzzy information. Inform. Sci. 180(5) (2010) 726- 736.

[15] D. J. Yu, Y. Y. $\mathrm{Wu}$ and T. Lu, Interval-valued intuitionistic fuzzy prioritized operators and their application in group decision making, Knowl-Based Syst. 30 (2012) 57-66.

[16] G. Beliakov, H. Bustince, D. P. Goswami, U. K. Mukherjee and N. R. Pal, On averaging operators for intuitionistic fuzzy sets, Inform. Sci. 181(6) (2011) 1116-1124.

[17] Z. S. Xu, Methods for aggregating interval-valued intuitionistic fuzzy information and their application to decision making, Control De. 22(2) (2007) 215-219. 
[18] Z. S. Xu and J. Chen, On geometric aggregation over interval-valued intuitionistic fuzzy information, in: The $4^{\text {th }}$ FSKD'07, Haikou, China, 2007, pp. 466-471.

[19] T. Y. Chen, H. P. Wang and Y. Y. Lu, A multicriteria group decision-making approach based on intervalvalued intuitionistic fuzzy sets: A comparative perspective, Expert Syst. Appl. 38(6) (2011) 7647-7658.

[20] S. M. Chen, M. W. Yang, S. W. Yang, T. W. Sheu and C. J. Liau, Multicriteria fuzzy decision making based on interval-valued intuitionistic fuzzy sets, Expert Syst. Appl. 39(15) (2012) 12085-12091.

[21] Z. J. Wang and K. W. Li, An interval-valued intuitionistic fuzzy multi-attribute group decision making framework with incomplete preference over alternatives, Expert Syst. Appl. 39(18) (2012) 13509- 13516.

[22] Z. L. Yue and Y. Y. Jia, An application of soft computing technique in group decision making under interval-valued intuitionistic fuzzy environment, Appl. Soft Comput. 13(5) (2013) 2490-2503.

[23] Y. J. Zhang, P. J. Ma, X. H. Su and C. P. Zhang, Multi-attribute group decision making under interval-valued intuitionistic fuzzy environment, Acta Autom. Sin. 38(2) (2012) 220-227.

[24] J. Y. Chai, J. N. K. Liu and Z. S. Xu, A rule-based group decision model for warehouse evaluation under interval-valued intuitionistic fuzzy environments, Expert Syst. Appl. 40(6) (2013) 1959-1970.

[25] S. M. Chen and T. S. Li, Evaluating students' answerscripts based on interval-valued intuitionistic fuzzy sets, Inform. Sci. 235 (2013) 308-322.

[26] G. İntepe, E. Bozdag, T. Koc, The selection of technology forecasting method using a multi-criteria interval-valued intuitionistic fuzzy group decision making approach, Comput. Ind. Eng. 65(2) (2013) $277-285$.

[27] K. Xu, J. Z. Zhou, R. Gu and H. Qin, Approach for aggregating interval-valued intuitionistic fuzzy information and its application to reservoir operation, Expert Syst. Appl. 38(7) (2011) 9032-9035.

[28] Z. H. Zhang, J. Y. Yang, Y. P. Ye, Y. Hu and Q. S. Zhang, A type of score function on intuitionistic fuzzy sets with double parameters and its application to pattern recognition and medical diagnosis, Procedia Eng. 29 (2012) 4336-4342.

[29] D. Kahneman, A. Tversky, Prospect theory: an analysis of decision under risk, Econometrica 47(2) (1979) 263-292.

[30] R. A. Krohling and T. T. M. de Souza, Combining prospect theory and fuzzy numbers to multi-criteria decision making, Expert Syst. Appl. 39 (2012) 11487-11493.

[31] S. Dhami and A. Al-Nowaihi, Why do people pay taxes? Prospect theory versus expected utility theory, J. Econ. Behav. Organization 64(1) (2007) 171-192.

[32] Z. P. Fan, X. Zhang, F. D. Chen and Y. Liu, Multiple attribute decision making considering aspiration-levels: A method based on prospect theory, Comput. Ind. Eng. 65(2) (2013) 341-350.

[33] G. Gurevich, D. Kliger and O. Levy, Decision-making under uncertainty - A field study of cumulative prospect theory, J. Bank. Fin. 33(7) (2009) 1221-1229.
[34] L. F. A. M. Gomes and M. M. P. P. Lima, TODIM: Basics and application to multicriteria ranking of projects with environmental impacts, Foundations Comput. Dec. Sci. 16(4) (1992) 113-127.

[35] V. Lotfi and T. J. Stewart, An aspiration-level interactive model for multiple criteria decision making, Comput. Ind. Eng. 19(7) (1992) 671-687.

[36] K. T. Atanassov, intuitionistic fuzzy sets, Fuzzy Set. Syst. 20(1) (1986) 87-96.

[37] S. K. De, R. Biswas and A. R. Roy, Some operations on intuitionistic fuzzy sets, Fuzzy Set. Syst. 114(3) (2000) 477-484.

[38] M. Abdellaoui, Parameter-free elicitation of utility and probability weighting functions, Manage. Sci. 46(11) (2000) 1497-1512

[39] H. T. Banks and M. Q. Jacobs, A differential calculus for multifunctions, J. Math. Anal. Appl. 29(2) (1970) 46-272.

[40] L. Stefanini, A generalization of Hukuhara difference and division for interval and fuzzy arithmetic, Fuzzy Set. Syst. 161(11) (2010) 1564-1584.

[41] Z. S. Xu and X. Q. Cai, Nonlinear optimization models for multiple attribute group decision making with intuitionistic fuzzy information, Int. J. Intell. Sys. 25(6) (2010) 489-513.

[42] F. Y. Meng, C. Q. Tan and Q. Zhang, The induced generalized interval-valued intuitionistic fuzzy hybrid Shapley averaging operator and its application in decision making, Knowl-Based Syst. 42 (2013) 9-19.

[43] X. M. Zhang, Z. S. Xu and X. H. Yu, Shapley value and Choquet integral-based operators for aggregating correlated intuitionistic fuzzy information, Information-TOYOKO 14(5) (2011) 1847-1858.

[44] L. S. Shapley, A value for n-person game, Princeton University Press, Princeton, 1953.

[45] M. Sugeno, Theory of fuzzy integral and its application, Doctorial Dissertation, Tokyo Institute of Technology, 1974.

[46] C. Q. Tan and X. H. Chen, Intuitionistic fuzzy Choquet integral operator for multi-criteria decision making, Expert Syst. Appl. 37(1) (2010) 149-157.

[47] J. Ye, Multicriteria fuzzy decision-making method based on a novel accuracy function under interval-valued intuitionistic fuzzy environment, Expert Syst. Appl. 36(3) (2009) 6899-6902.

[48] F. Y. Meng and J. Tang, Interval-valued intuitionistic fuzzy multi-criteria group decision making based on cross entropy and Choquet integral, Int. J. Intell. Syst. 28(12) (2013) 1172-1195.

[49] H. Bustince, M. Galar, B. Bedregal, A. Kolesárová and R. Mesiar, A new approach to interval-valued Choquet integrals and the problem of ordering in interval-valued fuzzy sets applications, IEEE Tran. Fuzzy Syst. 21(6) (2013) 1150-1162.

[50] H. Bustince, J. Fernández, A. Kolesárová and R Mesiar, Generation of linear orders for intervals by means of aggregation functions, Fuzzy Sets Syst. 220(1) (2013) 69-77. 